\title{
More than 160 near Earth asteroids observed in the EURONEAR network ${ }^{\star} \star \star$
}

\author{
M. Birlan ${ }^{1}$, O. Vaduvescu ${ }^{2,1,12}$, A. Tudorica ${ }^{4,16}$, A. Sonka ${ }^{6}$, A. Nedelcu ${ }^{3,1}$, A. Galad ${ }^{7,8}$, F. Colas ${ }^{1}$, F. Pozo N. ${ }^{12}$, \\ A. Barr D. ${ }^{12}$, R. Toma ${ }^{4,5,15}$, I. Comsa ${ }^{11}$, P. Rocher ${ }^{1}$, V. Lainey ${ }^{1}$, D. Vidican ${ }^{14}$, D. Asher ${ }^{10}$, C. Opriseanu ${ }^{14}$, C. Vancea ${ }^{4}$, \\ J. P. Colque ${ }^{12}$, C. P. Soto ${ }^{9}$, R. Rekola ${ }^{13}$, and E. Unda-Sanzana ${ }^{12}$
}

1 IMCCE, Observatoire de Paris, 77 avenue Denfert-Rochereau, 75014 Paris Cedex, France e-mail: mirel.birlan@imcce.fr

2 Isaac Newton Group of Telescopes, La Palma, Canary Islands, Spain

3 The Astronomical Institute of the Romanian Academy, Str. Cuţitul de Argint 5, 040557 Bucharest, Romania

4 University of Bucharest, Department of Physics, Platforma Măgurele, Str. Fizicienilor nr. 1, CP Mg - 11, Bucharest-Magurele 76900, Romania

5 The Romanian Society for Meteors and Astronomy (SARM), Târgoviște, Romania

6 Astronomical Observatory "Admiral Vasile Urseanu”, Bd. Lascăr Catargiu, nr. 21, sect. 1, Bucharest, Romania

7 Astronomical Institute, Academy of Sciences of the Czech Republic Fricova 1, 25165 Ondrejov, Czech Republic

8 Modra Observatory, Department of Astronomy, Physics of the Earth and Meteorology, FMFI UK, Bratislava 84248, Slovakia

9 Departamento de Ingeniería de Sistemas y Computación, Universidad Católica del Norte, Avenida Angamos 0610, Antofagasta, Chile

10 Armagh Observatory, College Hill, Armagh BT61 9DG, UK

11 Babes-Bolyai University, Faculty of Mathematics and Informatics, Str. Mihail Kogălniceanu nr. 1, 400084 Cluj-Napoca, Romania

12 Instituto de Astronomía, Universidad Católica del Norte, Avenida Angamos 0610, Antofagasta, Chile

13 Tuorla Observatory, Department of Physics and Astronomy, University of Turku, Finland

14 Astroclubul Bucureşti, Bd. Lascăr Catargiu, nr. 21, sect. 1, Bucharest, Romania

15 Rheinische-Friedrich-Wilhelms Universitaet Bonn Argelander Institut fuer Astronomie Regina-Pacis-Weg 3, 53113 Bonn, Germany

16 Institute for Space Sciences, Bucharest - Magurele 077125, Romania

Received 10 July 2009 / Accepted 16 November 2009

\section{ABSTRACT}

Context. The EUROpean Near Earth Asteroid Research (EURONEAR) is a network which envisions to bring some European contributions into the general context traced by the Spaceguard Foundation which was carried out during the last 15 years mainly by the US with some modest European and amateur contributions.

Aims. The number of known near Earth asteroids (NEAs) and potentially hazardous asteroids (PHAs) has increased tremendously, mainly thanks to five major surveys all focused on the discovery of new bodies. But also other facilities are required to follow-up and improvement the orbital parameters and to study the physical properties of the known bodies. These goals are better achieved by a co-ordinated network such as EURONEAR.

Methods. Astrometry is mandatory in order to acquire the positional information necessary to define and improve orbits of NEAs and PHAs and to study their trajectories through the solar system, especially in the vicinity of Earth. Photometry is required to derive some physical information about NEAs and PHAs. In order to achieve these objectives, the main method of research of the EURONEAR is the follow-up programme of objects selected by a few criteria, carried out mostly at $1 \mathrm{~m}$-class telescopes endowed with medium and large field cameras.

Results. 162 NEAs summing more than 1500 individual positions were observed for a total time of 55 nights in both visiting mode and regular runs using nine telescopes located in four countries. The observations were reduced promptly and reported to the Minor Planet Centre (MPC) which validated and included them in the MPC and NEODyS databases following the improvement of their orbital elements. For one binary NEA we acquired photometry and were able to determine its orbital and rotational periods. Complementary to the follow-up work, as many as 500 unknown moving objects consistent with new Main Belt asteroids and one possible NEA were discovered in the analyzed fields.

Conclusions. Our positions present $1^{\prime \prime}$ precision with an accuracy of $0.2-0.4^{\prime \prime}$, sufficient for achieving our immediate main goals. The observations and data reduction were conducted by our network members, which included some students and amateurs supervised by professional astronomers. In most cases, we increased the observational arcs decreasing the uncertainties in the orbits, while in some cases the new positions allowed us to recover some bodies endangered to be lost, defining their orbits.

Key words. astrometry - minor planets, asteroids: general

^ Based on observations acquired in Pic du Midi, Haute Provence, La Silla, Cerro Tololo, Las Campanas, Cerro Armazones, Bucharest Urseanu, and York University Observatories.

$\star \star$ Astrometric and photometric data are only available in electronic form at the CDS via anonymous ftp to cdsarc.u-strasbg.fr (130.79.128.5) or via

http://cdsweb.u-strasbg.fr/cgi-bin/qcat?J/A+A/511/A40 


\section{Introduction}

Near Earth asteroids (NEAs) are designated as small bodies of the solar system with a perihelion distance $q \leq 1.3$ AU and aphelion distances $Q \geq 0.983 \mathrm{AU}$ (Morbidelli et al. 2002). Potentially hazardous asteroids (PHAs) are designated as NEAs having a minimum orbital intersection distance $(M O I D) \leq 0.05 \mathrm{AU}$ (Bowell \& Muinonen 1994; Milani et al. 2002) and the absolute magnitudes $H \leq 22$, which corresponds to objects larger than about $150 \mathrm{~m}$, assuming an albedo of $13 \%$. Among the PHAs we can distinguish the virtual impactors (VIs), objects for which the estimation of impact probability ${ }^{1}$ with the Earth is non-zero (Milani et al. 2000a,b).

The interest in the study of NEAs increased tremendously during the last two decades, as the consciousness of the potential danger of these objects for the human society has been raised. Following Milani et al. (2002), the importance of knowledge about NEAs population for the human society was marked in 1990 by the US Congress. NASA was charged with the organization of a strategy "to study ways of increasing the discovery rate of these objects as well as to study the technologies and options for deflecting or destroying an $\mathrm{NEO}^{2}$, should it be found to pose a danger to life on Earth". The first report on this subject (Morrison 1992) proposed an international NEO survey programme called Spaceguard.

In the last decade we assisted to a complete a list of large sized NEAs/PHAs $(D>1 \mathrm{~km})$. This situation is due mainly to five NEAs discovery programs in progress, mostly carried out in the USA: Catalina Sky Survey ${ }^{3}$ (Larson et al. 1999; Stokes et al. 2002), LINEAR (Viggh et al. 1998), Spacewatch (Gehrels \& Jedicke 1996), LONEOS (Howell et al. 1996), and NEAT (Helin et al. 1997).

In the mean time, the interest in NEAs could be noticed in a few observatories ${ }^{4}$ across Europe, and we introduce three of them here.

Klet' Observatory programme in the Czech Republic has been focused on the follow-up tasl using a $0.57 \mathrm{~m}$ telescope equipped with CCD cameras (Tichá et al. 2000). About 600 NEAs, summing more than 6500 individual positions were reported between 1994 and 1999. Based on this experience, their new KLENOT programme, using a $1.06 \mathrm{~m}$ telescope (Tichá et al. 2002) is currently working and reports follow-up astrometry for about 2700 NEAs observed between 1998 and 2008 and three NEA discoveries.

Another important program we used for this was CINEOS (Bernardi et al. 2002). This program started regular observations on NEAs in 2001, using the 0.60/0.90 m Schmidt telescope of Campo Imperatore Observatory, Italy. The CINEOS survey was designed to discover new objects, joining the main goal of Spaceguard, namely to discover more than $90 \%$ of kilometersized NEOs by the end of 2008. Between 2001 and 2005, this program reported 30000 astrometric positions and was credited with the discovery of seven NEAs (Boattini et al. 2007).

The most prolific NEA discovery program in Europe appears to be carried out at the Crni Vrh observatory in Slovenia, and is actually run by a team of amateur and professional

\footnotetext{
${ }_{1}$ Milani et al. (2000b) underline the difference between the possibility of an impact with the Earth from the calculus of the probability of impact.

2 Near Earth Object.

3 Acronym CSS in the article.

${ }^{4}$ In our knowledge there is no exhaustive statistics at this moment in the World (in general, and in Europe in particular) for NEAs activities.
}

astronomers $^{5}$. Equipped with a robotic $60-\mathrm{cm}$ telescope controlled over the internet since 2004, this program leads the European contributions with 13 NEA discoveries (J. Skvarc 2009, private communication).

Astrometry of NEAs is also performed in Russia (Devyatkin et al. 2007), and jointly in Turkey and the Ukraine (Ivantsov et al. 2008), but their programs are not formalized with specific names.

The International Astronomical Union sponsored data centers ${ }^{6}$ which are open to all observational reports over the world. These reports are to follow rigorous steps for the validation of the acquired astrometric data (newly discovered or not) before being used in the calculus of orbital elements. New rules are also proposed for increasing the standard procedure (Milani et al. 2007).

It is important to explain the fundamental difference between a program dedicated to the discovery of NEAs and and the one devoted to the follow-up, securement and improvement of orbital elements. In a general sense, the requirement of a survey is to scan a large part of the sky during the entire observing night and discover as many NEAs as possible. Nevertheless, most of the survey programs use instruments with large pixel sizes, sometimes up to $3^{\prime \prime}$, which is inappropriate for accurate astrometry. Alternatively, a follow-up and recovery program focuses on accurate astrometric observations of selected objects announced in the alerts and observing lists maintained by dedicated authorities (such are MPC, Spaceguard Center, JPL, etc.). The requirements of such a follow-up and recovery program are shifted to observations of relatively small fields $\left(10 \div 30^{\prime}\right)$ and an instrument with a small pixel size $\left(0.3 \div 1.0^{\prime \prime} / \mathrm{pixel}\right)$.

Follow-up observations of NEAs/PHAs are necessary for several important reasons; namely i) once a new NEA is discovered, immediate observations are necessary to recover and secure the knowledge of its orbit; ii) once a PHA is determined, follow-up observations are necessary in order to improve its orbit, to be able to predict future close encounters and possible collisions with Earth; iii) studies of its physical parameters such as rotation periods, color, albedo, taxonomy and size are necessary to extract information about the most possible encounters.

The EURONEAR project envisions to establish a co-ordinated network which will follow-up and recover NEAs/PHAs using 1-2 m-class telescopes (dedicated and non-dedicated) located in Europe and elsewhere (Vaduvescu et al. 2008). While the programs CINEOS, KLENOT, and Crni Vrh are mainly focused on using specific telescopes in Europe to perform observations, the major objective of EURONEAR has been the high precision astrometry of NEAs in a co-ordinated network and the creation of synergies between European planetologists (and not only) for follow-up, precovery, and recovery of NEAs and PHAs.

EURONEAR has been working since May 2006. Besides its main scientific interest, the EURONEAR network is organized in order to increase the collaboration, mostly European, between professional astronomers, amateurs and students interested in NEAs. The implementation of the network is organized around a dynamic EURONEAR website and a specific list of emails.

\footnotetext{
5 http://www.observatorij.org/

6 AstDys, MPC.
} 
M. Birlan et al.: More than 160 NEAs in the EURONEAR network

Table 1. Telescopes and detectors used for follow-up, recovery and secure orbits of NEAs.

\begin{tabular}{lclccc}
\hline \hline Telescope & Aperture $(\mathrm{m})$ & CCD & Pixels & FOV $(\operatorname{arcmin})$ & Scale $\left({ }^{\prime \prime} /\right.$ pixel $)$ \\
\hline Pic du Midi & 1.05 & THX7863 & $384 \times 288$ & $5.2 \times 3.9$ & 0.820 \\
Haute Provence & 1.20 & TK1024 & $1024 \times 1024$ & $11.7 \times 11.7$ & 0.685 \\
Cerro Armazones & 0.85 & SBIG ST-10 & $2184 \times 1472$ & $15.6 \times 8.3$ & 0.430 \\
Cerro Tololo & 1.0 & Y4KCam & $4096 \times 4096$ & $20 \times 20$ & 0.289 \\
Las Campanas & 1.02 & SIT\#3 & $2048 \times 3650$ & $15.1 \times 26.5$ & 0.435 \\
La Silla & 1.00 & SBIG ST-10 & $2184 \times 1472$ & $3.3 \times 2.2$ & 0.090 \\
La Silla & 2.20 & WFI mosaic & $8 \times(2184 \times 1472)$ & $34 \times 33$ & 0.238 \\
Vasile Urseanu, & & & & & \\
Bucharest & 0.30 & TC273-home made & $640 \times 500$ & $21.6 \times 16.9$ & 2.30 \\
York University & 0.60 & SBIG ST-9 & $512 \times 512$ & $4.3 \times 4.3$ & 0.505 \\
\hline
\end{tabular}

Notes. The observatory is given in the first column and the aperture in the second one. The CCD camera (third column), the pixel size (fourth column), the field of view (FOV), and the scale (in "/pixel) are also presented.

\section{Observations and astrometry}

\subsection{Observations}

A total of 55 nights of observations are reported in this article. Most of the observations were obtained in visiting mode at several observatories across the world. These runs were partly obtained as the result of regular semestrial applications for observing time. The proposals submitted were successful, and we observed in this mode for a total time of 22 nights. The rest of the nights were observed with the telescopes in Pic du Midi, Cerro Armazones, Admiral "Vasile Urseanu" in Bucharest, and York University.

The runs obtained via regular applications offer the advantage of having enough time for preparing the run and the ad hoc team to work for data reduction. This organization is essential when a huge amount of data is obtained during the run (for example the run with $2.2 \mathrm{~m} \mathrm{MPI/WFI} \mathrm{on} \mathrm{La} \mathrm{Silla).} \mathrm{On} \mathrm{the} \mathrm{other}$ hand, the facilities at disposal offer more flexibility of schedule, allowing among other things for the assessment of specific newly released targets which may need urgent observation. We used a total of nine telescopes located in four countries: France, Chile, Canada and Romania. These facilities are presented in Table 1.

NEAs are objects with fast apparent movement, with a period of visibility which can change dramatically in just a few days. For that, EURONEAR has developed its own scheduler program for NEA observations, available on its webserver ${ }^{7}$. For newly discovered objects, the optimum window of observations is defined for each asteroid according to a few factors, so as to ensure maximum probability of successful follow-up and consequently securing of their orbits. Typically, windows of about 20 days after discovery suffice in such cases. This scheduling tool is at the disposal of the scientific community.

We used a binning mode of $3 \times 3$ for the ESO/MPG $2.2 \mathrm{~m}$ and ESO $1 \mathrm{~m}$ telescopes due to requirements in minimizing the data transfer and oversampling. The atmospheric conditions were quite heterogeneous between several sites, with the average seeing estimations in the range of $0.8 \div 4.0^{\prime \prime}$, the lowest value being in La Silla and Las Campanas, while the worst seeing was recorded for the Vasile Urseanu observatory in Bucharest, and York University in Toronto.

\subsection{Astrometry}

The images were calibrated by flat field and bias frames before the astrometric measurements. Approximately $80 \%$ of the

\footnotetext{
7 http://euronear.imcce.fr
}

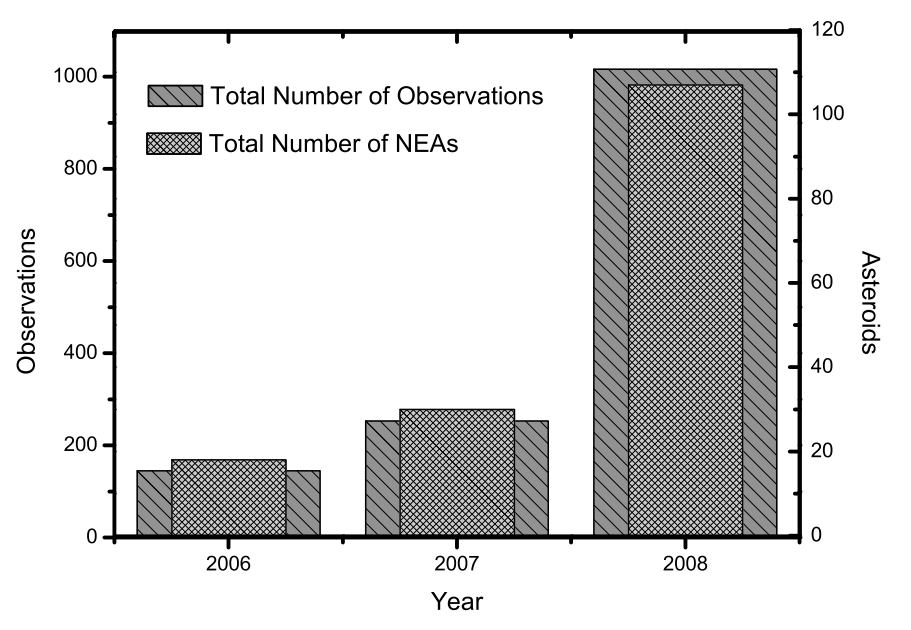

Fig. 1. The 1538 positions of a total of 162 NEAs observed in the EURONEAR network, in cumulative number per year, between 2006 and 2008 .

images were reduced using the Astrometrica software ${ }^{8}$ and the USNO-B1 catalog. Astrometrica is easily accessible and very often used by the amateur community for asteroid observations, and we decided to use it mainly because most of our data reduction was made by students and amateurs, members of our network. Despite its relatively low astrometric precision and lack of proper motion of sources, we preferred the USNO-B1 versus UCAC2 catalog because of the higher density of stars of USNOB1 and its all-sky coverage, thus the astrometric calibration of small fields is always possible.

For the rest of the images we used some personal procedures in MIDAS, IDL or IRAF. It is important to note that some of the observations were treated with a specific software ${ }^{9}$ which is under development among the EURONEAR members, based on the CPL ${ }^{10}$ procedures and using the UCAC2 catalog.

162 NEAs, summing more than 1500 individual positions were observed in the visiting mode in the frame of the EURONEAR network, between May 2006 and Dec 2008, as presented in Fig. 1. The observing log table is presented in the Appendix of this article and includes the telescope location, the name of the asteroid, the type, the date of observations,

\footnotetext{
8 Raab, H. 2008, http : //www . astrometrica . at

9 The embryo of a future pipeline to be used by our network.

10 http://www . eso.org/sci/data-processing/software/ $\mathrm{cpl} /$
} 
Table 2. An example of the catalog EURONEAR_Release1.

\begin{tabular}{ccccccc}
\hline \hline Asteroid & $\begin{array}{c}\text { Date } \\
\text { UT }\end{array}$ & $\begin{array}{c}\text { Right ascension } \\
(\mathrm{h} \mathrm{m} \mathrm{s})\end{array}$ & $\begin{array}{c}\text { Declination } \\
\left({ }^{\prime \prime}{ }^{\prime \prime}\right)\end{array}$ & Mag & Filter & Obs code \\
\hline 162173 & 20080312.02639 & 062935.020 & -135802.20 & 18.2 & $R$ & 809 \\
162173 & 20080312.02795 & 062935.540 & -135801.10 & 18.1 & $R$ & 809 \\
162173 & 20080312.02970 & 062936.110 & -135800.00 & 18.1 & $R$ & 809 \\
162173 & 20080312.03134 & 062936.670 & -135758.90 & 18.1 & $R$ & 809 \\
162173 & 20080312.03314 & 062937.270 & -135757.70 & 18.1 & $R$ & 809 \\
162173 & 20080312.03509 & 062937.930 & -135756.40 & 18.2 & $R$ & 809 \\
162173 & 20080312.03672 & 062938.460 & -135755.40 & 18.0 & $R$ & 809 \\
162173 & 20080313.03905 & 063529.610 & -134654.10 & 17.9 & $R$ & 809 \\
162173 & 20080313.04053 & 063530.110 & -134653.10 & 17.9 & $R$ & 809 \\
162173 & 20080313.04209 & 063530.620 & -134652.00 & 17.8 & $R$ & 809 \\
162173 & 20080313.04562 & 063531.790 & -134649.50 & 17.9 & $R$ & 809 \\
162173 & 20080313.04877 & 063532.850 & -134647.40 & 17.8 & $R$ & 809 \\
162173 & 20080313.05032 & 063533.350 & -134646.30 & 17.9 & $R$ & 809 \\
162173 & 20080313.05196 & 063533.890 & -134645.20 & 17.9 & $R$ & 809 \\
162173 & 20080313.05358 & 063534.430 & -134644.00 & 17.9 & $R$ & 809 \\
\hline
\end{tabular}

Notes. Asteroid number (or its provisory designation), date, right ascension, declination, magnitude, filter, and observatory code are presented.

the apparent magnitude, the exposure time, and the number of observations. This table contains also the apparent motion of the asteroid and the time period (d-days, y-years) between its discovery and the EURONEAR observation.

Most of the runs were conducted using sidereal tracking. However, for a few runs (Pic du Midi and ESO/MPG) we tracked using the half-value of the proper motion of the observed asteroids, which has the advantage to have shorter trails for the asteroids while increasing the exposure time in order to reach fainter magnitudes.

To reduce the ESO/WFI images, a specific procedure was developed. Following the flat and bias treatement of the images, we sliced the mosaic image in the eight CCD components which were treated independently, and we measured them separately. To take advantage of the large field of the ESO/MPG, $\mathrm{LCO} /$ Swope and CTIO $1 \mathrm{~m}$ runs, we inspected the entire field of these runs besides the observed NEA and reported all asteroids (known and new) found in the images.

Depending on the field of the telescope and the density of the stars in the field, the scale and the orientation of the field were computed using about 6 to 100 catalog stars with a precision better than $0.3^{\prime \prime}$. The measured $(x, y)$ values were related to the standard $(X, Y)$ coordinates using a linear model. Typically, the differences between the measurements and the catalog positions do not exceeded 300 mas.

The center $(x, y)$ of the measured sources was obtained using a 2D-symetric Gaussian function. The rms fit of the center was lower than 150 mas, depending on the telescope aperture, the magnitude, the seeing, and the method of guiding during the acquision of the image.

\section{Results}

The catalog EURONEAR_Release 1 contains all the data referred into the article. The columns of the release are: the name of the asteroid, rigth ascension and declination for the J2000.0 epoch, magnitude and filter (when available) and the UAI observatory code. A sample of the release is presented in Table 2.

References of reported EURONEAR astrometry are in the following Minor Planets (Electronic) Circulars: Pozo et al. (2008); Scotti et al. (2008a,b); Cavadore et al. (2008); Kern et al. (2008); Elst et al. (2008c); Buie et al. (2008); Vaduvescu \& Tudorica (2008); Tubbiolo et al. (2008); Young et al. (2008); Elst et al. (2008a,b); Tholen et al. (2008b); Sheppard et al. (2008); Vaduvescu et al. (2007a,d,b,c); Cavadore et al. (2006).

\section{EURONEAR}

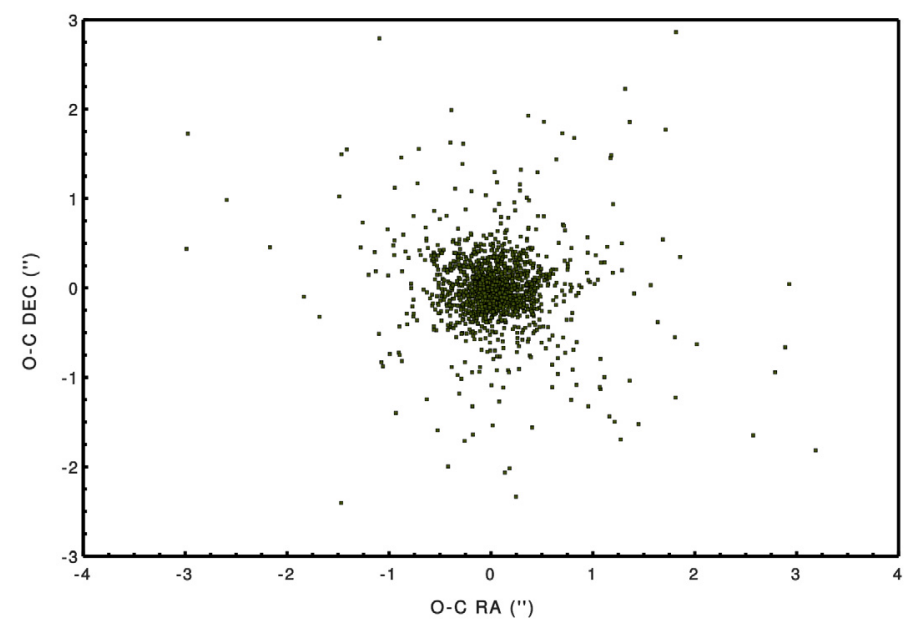

Fig. 2. (o-c) residuals for 1538 positions of 162 NEAs observed in the EURONEAR network. Most of the points are confined within 1", probing the observational capabilities for all facilities and the accurate data reduction.

All astrometric measurements were accepted by MPC, being included successfully in the procedure of adjustment of the orbital catalogs maintained by JPL and NEODyS. Figure 2 presents the distribution of observed minus calculated (o-c) positions in right ascension and declination for all runs. The values are mostly confined within $1^{\prime \prime}$ for all observatories, probing the precision of data reduction, observational capabilities and the available instrumentation. The frequencies of values (Fig. 4) are centered relatively close to zero.

The individual plots of the (o-c) values for each telescope are available in the Appendix of this paper. The dispersion of observations between observatories is different and is related to the telescope aperture, pixel size and seeing conditions. We will focus our interest on the telescopes for which the (o-c) values are well confined around the zero value.

The Gaussian fit was performed for all (o-c) values in right ascension and in declination, assuming non-weighting data, in free parameter mode. In the right ascension, the Gaussian distribution corresponds to $94.97 \%$ of the correlation and the $F W H M$ is $(0.401 \pm 0.019)^{\prime \prime}$, while for the declination the correlation in $97.81 \%$ and the $F W H M$ is $(0.431 \pm 0.013)^{\prime \prime}$. The 


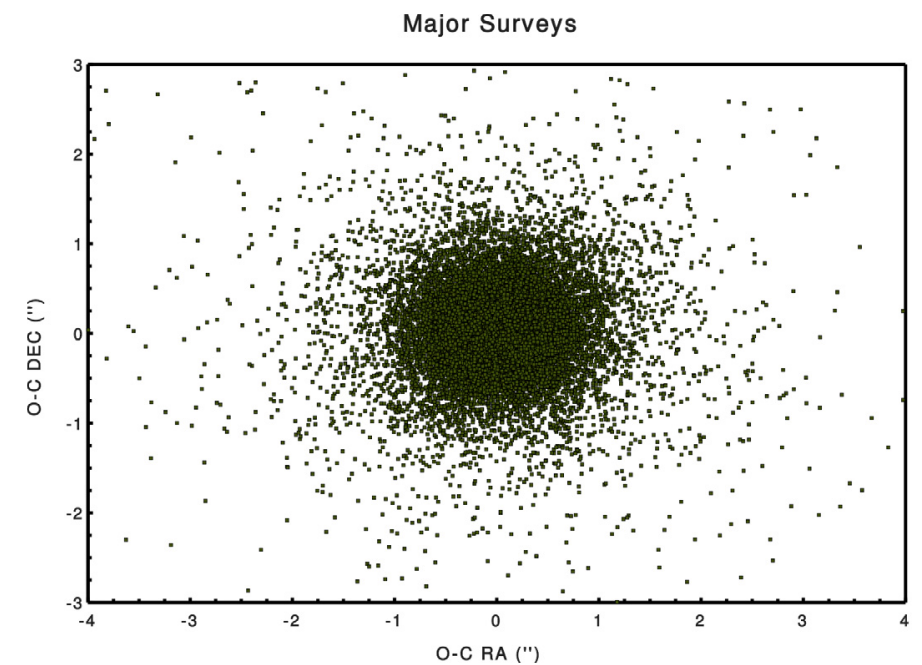

Fig. 3. Over 23000 (o-c) residuals related with observations performed by all other surveys which observed in the past the same asteroids with EURONEAR. Comparing this plot with the one of Fig. 2, one can observe that EURONEAR observations appear better confined around zero, and this fact is also supported by statistics.

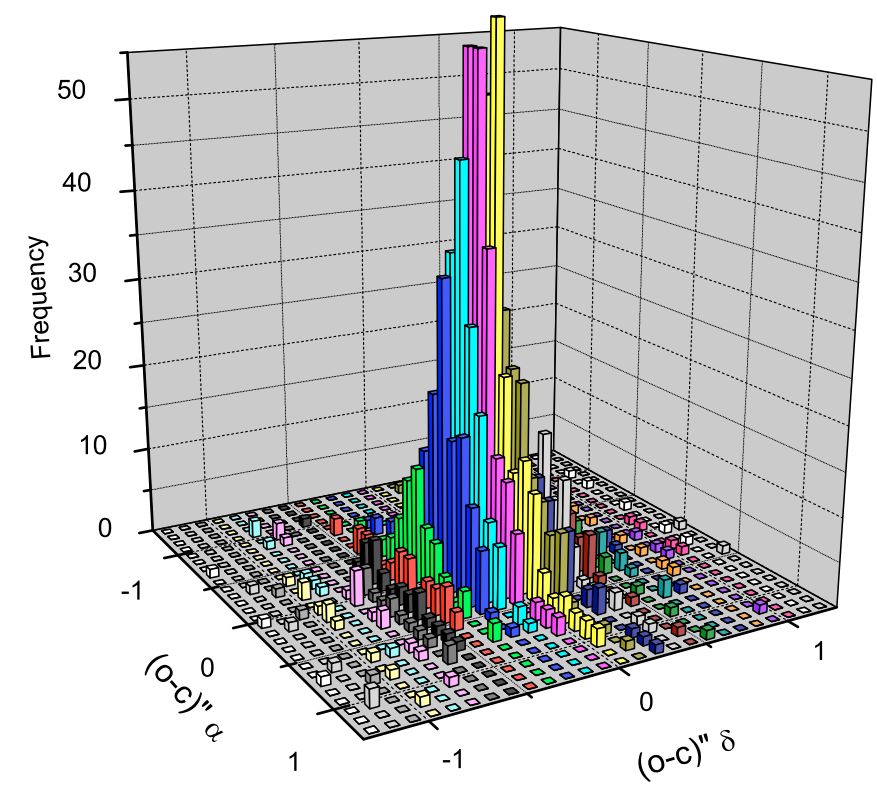

Fig. 4. The frequency of (o-c) values in the plane of right ascension and declination, with a bin value of $0.095^{\prime \prime}$. The values of frequency are centered to the zero value.

maximum of the Gaussian fit is slightly shifted to positive values, $(0.022 \pm 0.008)^{\prime \prime}$ in right ascension and $(0.042 \pm 0.006)^{\prime \prime}$ in declination.

The (o-c) values are very well centered around zero for the observations obtained with the $2.2 \mathrm{~m}$ telescope. The best Gaussian function is obtained for a $F W H M=(0.218 \pm 0.012)^{\prime \prime}$ centered at $(0.020 \pm 0.005)^{\prime \prime}$ in right ascension, and $F W H M=$ $(0.261 \pm 0.037)^{\prime \prime}$, centered at $(0.023 \pm 0.015)^{\prime \prime}$ in declination. These values show a very good consistency between the internal errors of the data and the expected position errors from the USNO-B1 reference catalog. Careful analysis of the data shows that the (o-c) values are widely spread for newly discovered objects whose orbit uncertainties were larger, while for the objects

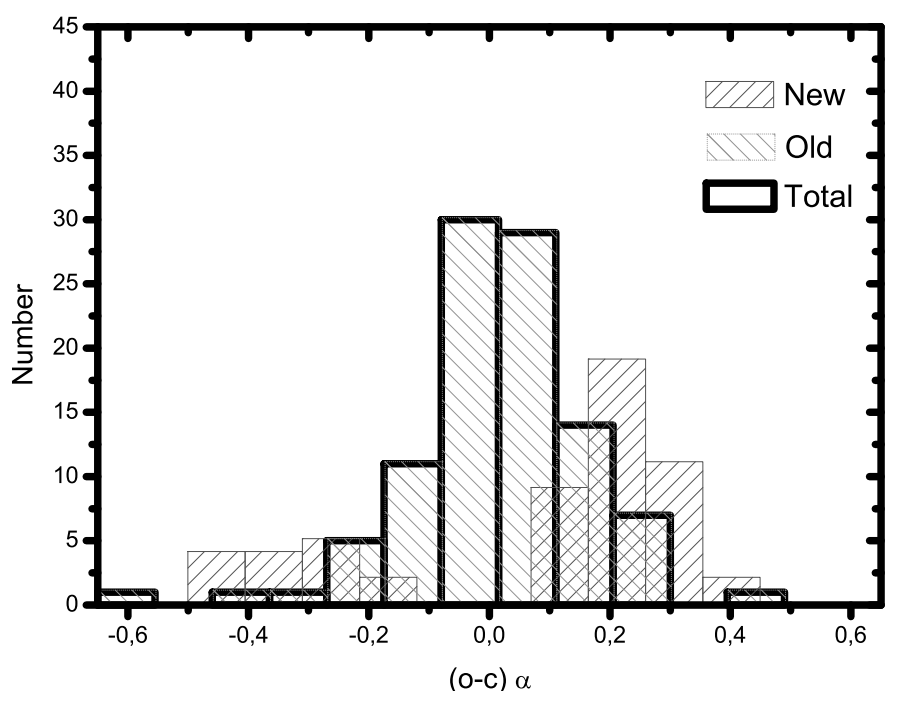

Fig. 5. (o-c) residuals in right ascension for the asteroids observed with $2.2 \mathrm{~m}$ telescope, La Silla. These values are widely spread for newly discovered objects whose orbit uncertainties were larger, while for the objects observed at two (or more) oppositions the values are well confined around zero.

observed at two (or more) oppositions the (o-c) values are well confined around zero. This could be seen in Fig. 5.

Recently, Tholen et al. (2008a) described systematic offsets in the astrometry of (99942) Apophis for more than 200 individual observations. This large offset of approximately $0.2^{\prime \prime}$ to the north of ephemerides position was associated to the systematic errors in declination of the star catalog ${ }^{11}$, compared to the ICRF sources. From our global histograms of observed objects we cannot confirm such a kind of offset, which suggests that the $0.2^{\prime \prime}$ error is related to a local systematic error in the catalog. Also, such a kind of analysis for a given asteroid of our sample is not possible, while for each object the number of individual observations is around ten (thus statistically irrelevant) and over a short arc of orbit.

We compared our work with other programs, mostly the five major US surveys capable to regularly observe NEAs using facilities similar to those used by our network. In Fig. 3 we plot (o-c)s derived from data taken by all other surveys for the same NEAs which were observed by us. Over 23000 (o-c) points of Fig. 3 refer to the same observed objects plotted in Fig. 2. Our data appear to be better confined around zero (about $0.4^{\prime \prime}$ ) than the (o-c) points from the other surveys (about 0.6"). Similar analysis carried out separately for each telescope used by our network arrives at similar results, with even better (o-c) confinement in the case of the ESO/MPG $2.2 \mathrm{~m}$ and LCO $1 \mathrm{~m}$ datasets.

\subsection{Recovered VIs, PHAs, and NEAs}

Besides NEAs selected from the Spaceguard and MPC object lists, we checked during a few runs also the MPC NEO confirmation list. Six minor planets were recovered by EURONEAR at OHP, CTIO, and LCO within one day from discovery, following their alert on the MPC list, while another four could not be recovered. The recovered objects are marked by $(*)$ in the Appendix.

\footnotetext{
11 They used USNO-B1.
} 
The recovered objects are: $2007 J X_{2}$ (MPEC 2007-J32) PHA discovered by Catalina and recovered by EURONEAR at OHP 1.2 m (formerly 7J2E987); $2008 K E_{6}$ (MPEC 2008-K63) - PHA discovered by Catalina and recovered by EURONEAR at CTIO 1 m (formerly 8K85BC5); $2008 K N_{11}$ (MPEC 2008-K66) - VI discovered by Catalina and recovered by EURONEAR at CTIO $1 \mathrm{~m}$ (formerly 8K86577); $2008 U Q_{2}$ (MPEC 2008-U45) MBA discovered by Catalina and recovered by EURONEAR at LCO $1 \mathrm{~m}$ (formerly 8UB57EF); $2008 U T_{2}$ (MPEC 2008-U48) - NEA discovered by Catalina and recovered by EURONEAR at LCO $1 \mathrm{~m}$ (formerly 8UB733D) and $2008 U R_{2}$ (MPEC 2008-U46) - NEA discovered by Spacewatch and recovered by EURONEAR at LCO $1 \mathrm{~m}$ (formerly SW40ea).

Part of the observed NEAs, EURONEAR observed one NEA having a very large sigma uncertainty, namely $2008 J A_{8}$, discovered by CSS on May 5, 2008, which was recovered by EURONEAR at CTIO $1 \mathrm{~m}$ about $10^{\prime}$ away from predicted position, later being tracked back and identified with a NEAT 2001 observation thanks to EURONEAR that defined its orbit.

\subsection{Astrometry and orbit adjustment: the case of 2008 $C R_{118}$ as a scholarship example}

The astrometry performed by EURONEAR contributes to the improvement of the orbital elements of NEAs. These elements are updated regularly by several services and teams (e.g. MPC, NEODyS, ASTORB). These new orbital elements are dependent on several factors, such as the initial conditions of the adjustment, the weight of individual observations, the procedure of the adjustment of observations, the precision (double, quadruple) in the procedure, the dynamical system used for the integration, and the choice of the numerical integrator.

It is important to note that the adjustment is mandatory for objects for which one has a poor knowledge of their orbital parameters. This occurs mainly in two situations: i) the object was not observed for a long time, and ii) the object is a new discovery.

We propose to investigate this problem in a non-exhaustive way for our whole sample and to provide an example of such new observations, thus underlining the importance of an orbital adjustment of NEAs. We will concentrate on the asteroid 2008 $C R_{118}$ as a generic example of the situation described below.

We computed the orbital elements of the NEA $2008 C R_{118}$ from 80 individual observations, obtained between March 2003 and October 2008. Ten of these astrometric observations were obtained by EURONEAR in Las Campanas (IAU code 304), on Oct. 22, 2008. When the EURONEAR observations were performed, the estimated uncertainty of the MPC ephemeris was around $20^{\prime \prime}$.

In our investigation we used two different approaches to quantify the importance of these new observations. The first approach which we used was NOE-AST, derived from Lainey et al. (2007), Lainey (2008), and Lainey \& Vienne (2009). This procedure uses the dynamical model DE406. The treshold of the adjustment in the procedure was established at $3 \sigma$, while the numerical integration was based on Gauss-Radau polynomials (Everhart 1985). The second approach, ASTERPRO (Rocher 2007; Bec-Borsenberger \& Rocher 1988), is a specific procedure of adjustment and orbital element computation developed by Patrick Rocher. This procedure uses the dynamical model SLP98 (G. Francou, personal communication), the post-newtonian correction, $\chi^{2}$ minimization procedure and $\mathrm{K}$-student test as well as the Bulirsch and Stoer numerical integrator.

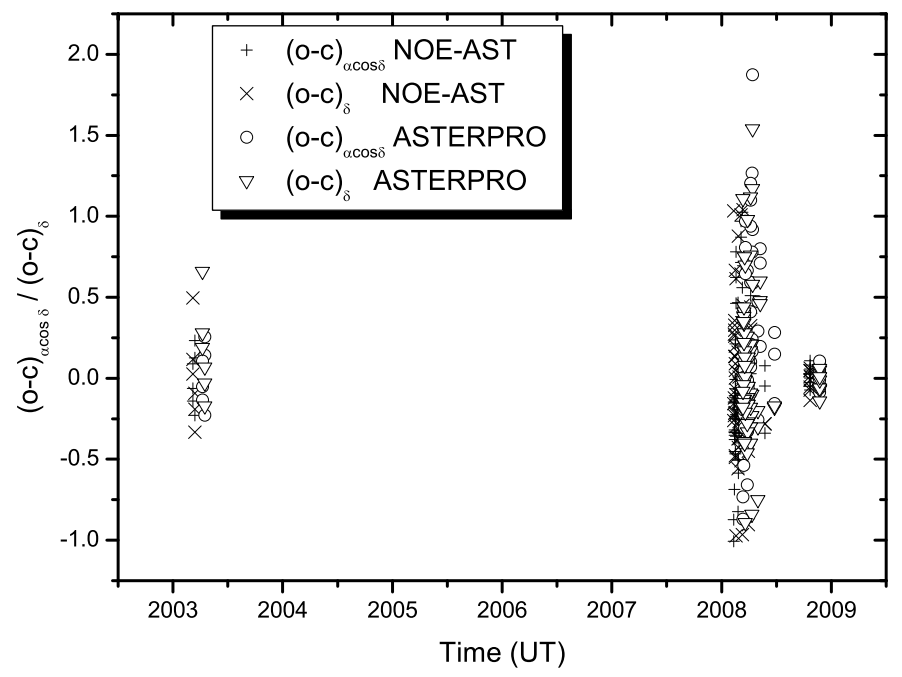

Fig. 6. The (o-c) values in right ascension and declination, correspondent to the adjustment of $2008 C R_{118}$ using NOE-AST and ASTERPRO.

We used the orbital elements for the epoch June 18, 2009, proposed by the Lowell Observatory ${ }^{12}$ as initial conditions in our adjustment, and the weight of each observation was considered equal to the unity (i.e. independent observations).

The reliability of the results after the adjustment was checked using several NEAs, by comparing the rectangular coordinates $X, Y$, and $Z$ and their first derivatives $\dot{X}, \dot{Y}$, and $\dot{Z}$, as well as the (o-c) values of the observations which were retained for the final solution. Globally the differences in rectangular coordinates are below $10^{-7}-10^{-6} \mathrm{AU}$, at values similar to the standard deviation of the observations. In the case of $2008 C R_{118}$, these values are $10^{-6}, 0.6 \times 10^{-6}$, and $10^{-7} \mathrm{AU}$ for $X, Y$, and $Z$ respectively (i.e. less than $1 \mathrm{~km}$ of difference). These differences correspond to excursions of semi-major axis of less than $10^{-7} \mathrm{AU}$ and less than $3 \times 10^{-7}$ in eccentricity. The correspondent (o-c) in right ascension and declination are presented in Fig. 6.

We applied these two methods of adjustment to the asteroid $2008 C R_{118}$ in two situations: i) the case of the observation sample on which we extracted the observations from October 22, 2008, (Obs-without-EURONEAR) and ii) the case of a sample including all observations (Total-Obs). Then, the comparison of results would give an estimation of the improvement in the orbital coordinates, in NOE-AST and ASTERPRO approach respectively.

The results of the differences in coordinates and velocities (Total-Obs minus Obs-without-EURONEAR) are presented in Table 3. We conclude an important influence of the observations of Oct. 22, 2008 in the cartesian coordinates of the asteroid. Thus, for the $X$ cartesian coordinate, the differences are $1995.6 \mathrm{~km}$ and $3383.1 \mathrm{~km}$ for NOE-AST and ASTERPRO respectively, which is more important by two orders than that for the $Y$ coordinate. Relatively important differences are also calculated for the $Z$ coordinate $(358.6 \mathrm{~km}$ and $446.8 \mathrm{~km}$ for NOE-AST and ASTERPRO respectively). Consequently, this is translated in differences of $10^{-7}$ in the semi-major axis and $0.8 \times 10^{-6}$ in eccentricity, for both the NOE-AST and ASTERPRO methods.

12 ftp://ftp.lowell.edu/pub/elgb/astorb.dat.gz 
M. Birlan et al.: More than 160 NEAs in the EURONEAR network

Table 3. Comparison of the differences of rectangular coordinates and velocities calculated for the asteroid $2008 C R_{118}$ using two adjustment procedures.

\begin{tabular}{lcccccc}
\hline \hline $\begin{array}{l}\text { Coordinate } \\
\text { MODEL }\end{array}$ & $\begin{array}{c}X \\
\left(\times 10^{-6} \mathrm{AU}\right)\end{array}$ & $\begin{array}{c}Y \\
\left(\times 10^{-6} \mathrm{AU}\right)\end{array}$ & $\begin{array}{c}Z \\
\left(\times 10^{-6} \mathrm{AU}\right)\end{array}$ & $\begin{array}{c}\dot{X} \\
\left(\times 10^{-7} \mathrm{AU} / \text { day }\right)\end{array}$ & $\begin{array}{c}\dot{Y} \\
\left(\times 10^{-7} \mathrm{AU} / \text { day }\right)\end{array}$ & $\begin{array}{c}\dot{Z} \\
\left(\times 10^{-7} \mathrm{AU} / \text { day }\right)\end{array}$ \\
\hline NOE-AST & 13.340 & -0.082 & 2.397 & 0.229 & 0.039 & -0.004 \\
ASTERPRO & 22.615 & -0.208 & 2.987 & -0.323 & 0.171 & 0.038 \\
\hline
\end{tabular}

Table 4. Sample of orbit adjustment (semi-major axis for briefness here) and MOID calculated with observational datasets available immediately before and after our observations, compared with the complete dataset available on July 6, 2009.

\begin{tabular}{lrrrrrr}
\hline \hline Asteroid & a before & a after & a present & MOID before & MOID after & MOID present \\
\hline 2006 KD1 & 1.610261 & 2.482955 & 2.483163 & 0.35501 & 0.41254 & 0.41225 \\
2008 KE6 & 0.815169 & 1.487399 & 1.685202 & 0.00177 & 0.01904 & 0.02243 \\
2008 KF6 & 2.279096 & 2.298772 & 2.325204 & 0.03458 & 0.03719 & 0.03864 \\
2008 KN11 & 1.733161 & 1.694478 & 1.708350 & 0.00101 & 0.00099 & 0.00099 \\
2008 UT2 & 1.795989 & 1.798609 & 1.804049 & 0.02341 & 0.02359 & 0.02357 \\
2008 EM7 & 1.230006 & 1.240259 & 1.238430 & 0.02553 & 0.01720 & 0.01864 \\
\hline
\end{tabular}

\subsection{Brief orbital study of VIs, PHAs, and recovered NEAs}

All together, EURONEAR observed 66 VIs, PHAs and recovered NEAs, according to their classifications available at the observing epoch (online Table). We considered these cases to fit the orbits and derive the semi-major axis (a) and Minimum Orbital Intersection Distances (MOID) from orbits fitted to the observational datasets available immediately before and after our observations, as well as those resulting from the fit of all data available at present (e.g., including all observations after our runs). To perform the orbital fitting we used the ORBFIT package ${ }^{13}$ and the asteroids' observational data from NEODyS ${ }^{14}$. Table 4 includes some results which show several important cases where the EURONEAR contribution can be seen.

\subsection{Photometry of NEAs}

Most NEAs observed by EURONEAR had magnitudes between $V=19 \div 20^{\mathrm{m}}$ (1 m class telescopes) as faint as $V=22^{\mathrm{m}}$ for the $2.2 \mathrm{~m}$ telescope (see Table in the Appendix), compatible with or complementing to other major surveys. Most observations were obtained using the Johnson or Cousins $R$ filter. In the case of faint asteroids, no filter was used to maximize the recording of the Sun flux reflected by the body.

One of the NEAs observed in Las Campanas, $1994 A W_{1}$, was actually the main target of a campaign focused on acquiring photometric data for a few selected binary asteroids (mostly Main Belt) to constrain the distribution of their orbital poles (Čapek \& Vokrouhlický 2004). A few weeks previous to our $1 \mathrm{~m}$ telescope observations in Chile, $1994 A W_{1}$ was observed using the $1 \mathrm{~m}$ telescope at SAAO in South Africa in poor weather conditions. By joining the two datasets we could obtain a better precision for the orbital and rotation periods.

A fast rotation period of the primary $1994 A W_{1}$ was derived, $P_{\text {syn }}=2.5193 \pm 0.0001 \mathrm{~h}$. The amplitude of the lightcurve was $0^{m} .17$. The orbital period of the system, $P_{\text {orb }}=22.38 \pm 0.01 \mathrm{~h}$, was obtained after realizing that only primary events (magnitude attenuation) were observed in February and in March 2008. The rotation period of the satellite coincides with its orbital period

\footnotetext{
13 http://adams.dm.unipi.it/ orbmaint/orbfit/

14 http://newton.dm.unipi.it/neodys/
}

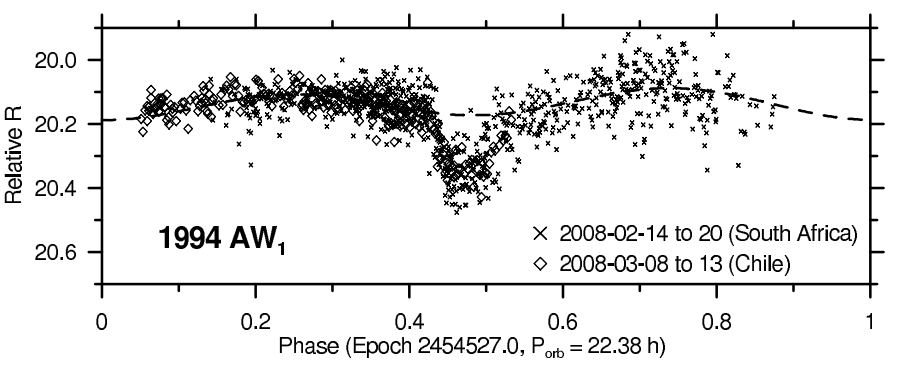

Fig. 7. Composite lightcurve of the binary system $1994 A W_{1}$ after subtraction of the short period. The dashed line is the assumed fit of the lightcurve, which does not cover the whole period.

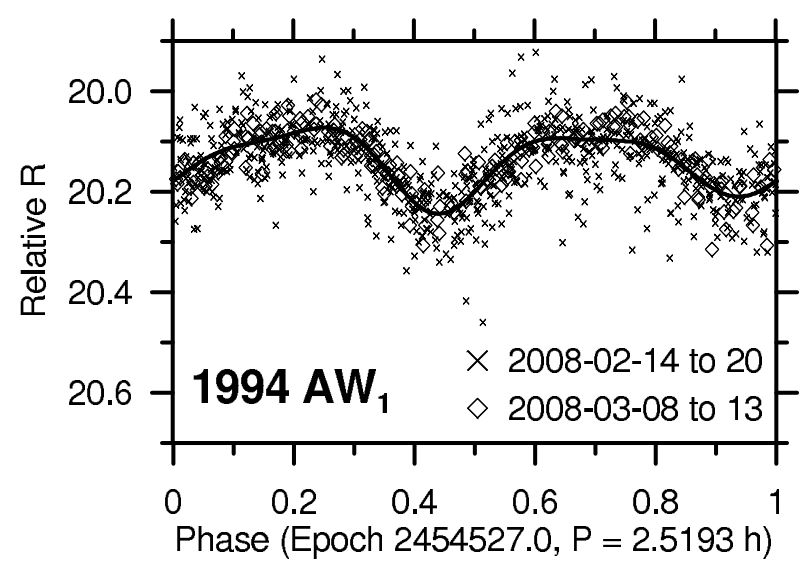

Fig. 8. The composite lightcurve of the primary body of the binary system $1994 A W_{1}$.

and the amplitude of the lightcurve (without fitted component from the rotation period of the primary) is at least 0 . 10 . The fits to the binary model in Fig. 7, which show the orbital period and in Fig. 8 the rotation period of the binary system. We compared the previous results of Pravec \& Hahn (1997) with ours, and the results are similar despite the change in the geometry of the system by about $50^{\circ}$ in longitude and $30^{\circ}$ in latitude (if one takes into account the so-called phase-angle bisector). 
Photometry and physical data of binary NEAs are rather sparse, mainly because photometry is a task very timeconsuming which requires a few nights or weeks to derive rotational and orbital periods for only a few systems. For most telescopes the observations for long periods seems to be incompatible with the policy of granted observing time. However, studies of physical properties of binary NEAs as well as their dynamical evolution are essential for the near future. In this regard, it is clear that a co-ordinated network to include at least two telescopes would be preferable, and EURONEAR could contribute in the near future with photometric data for NEA physical studies.

\subsection{Discovery of MBAs and NEAs}

Some of the NEAs followed-up by EURONEAR were located close to the ecliptic plane, and consequently many objects with proper motion compatible with MBAs could be identified in the observed fields. Besides the surveyed NEAs, data reducers closely inspected the entire fields, identifying and measuring all moving bodies using the convenient blink function of Astrometrica.

Most moving objects identified in the larger fields of $1 \mathrm{~m}$ telescopes (CTIO, LCO, OHP) were identified as known MBAs. Some others were not known and were detected in the runs at Las Campanas $1 \mathrm{~m}$ and ESO/MPG $2.2 \mathrm{~m}$, thanks to the larger FOV and larger aperture in the second case. All together, we identified, measured and reported to MPC some 50 unknown moving bodies in the LCO $1 \mathrm{~m}$ run (five nights in Oct $2008 \mathrm{ob}-$ serving some 50 fields covering about 6 sq. degree) and about 450 unknown bodies in the ESO/MPG run (Mar. 2008 observing some 42 WFI fields covering about 21 sq. degree). To date, 56 objects observed at ESO/MPG have been officially considered EURONEAR discoveries by the MPC.

One set of eight images observed at the ESO/MPG during one night shows a well-visible set of longer collinear trails (about 25 pixels each), which suggests an object moving quite fast (about $10 \mathrm{arcsec} / \mathrm{min}$ ), which could not be linked to any known NEA or PHA. We speculate that the accidental encounter of one new NEA during this three night run with ESO/MPG equipped with WFI mosaic camera could be compatible with the results of Boattini et al. (2004). In 2002 Boattini et al. (2004) discovered three NEAs during three clear nights using ESO/MPG at a time when the number of known NEAs was much less. Unfortunately, our object was observed for about 15 min only during one night, so it is impossible to establish an orbit, but it is expected to be linked to another object sometime soon. It is out of the scope of this paper to present our MBA discoveries, so we plan to investigate them statistically in the context of the MBA populations and $2 \mathrm{~m}$ survey capabilities at the faint end $\left(\operatorname{mag} V \approx 22^{\mathrm{m}}\right)$.

\section{Conclusions and perspectives}

EURONEAR network has been organized to increase the collaboration, mostly European, between professional astronomers, amateurs and students interested in NEAs. The optimization of this collaborative work of observations and astrometric data reduction is possible via a dedicated website which includes some specific software and a dedicated list of emails.

Astrometry has been the main tool of research for orbital properties. Overall, 162 NEAs, summing more than 1500 individual positions observed over a total of 55 nights between 2006 and 2008 were reported by the EURONEAR network and used in the orbital adjustment of NEAs (NEAs, PHAs, VIs). These results are published in the catalog EURONEAR_Release1. Some orbital fits run as examples with some models such as NOEAST, ASTERPRO, and ORBFIT including our datasets were compared with those obtained before our observations, clearly probing the contributions of our runs. Photometry is the main tool of research for basic physical properties of NEAs, and in this sense the NEA binary system $1994 A W_{1}$ was observed, and we derived its rotation and orbital period.

About 500 new objects, whose motions are compatible with MBAs were reported as potential serendipitous discoveries in Las Campanas $1 \mathrm{~m}$ and La Silla $2.2 \mathrm{~m}$, thanks to their large field and aperture capabilities. From these objects, 56 MBAs are officially recognized by the MPC as new discoveries. One object is speculated to be a new NEA which was possibly lost.

The (o-c) plots for all our observations show that most of our reduced data remained confined within $1^{\prime \prime}$, probing the observational capabilities for all facilities and the accurate data reduction. Considering our whole reduced dataset, the average astrometric position error has a $F W H M$ of $0.4^{\prime \prime}$ consistent with the USNO-B1 catalog uncertainty. This value is two times lower for the ESO/MPG observations, which benefit by the $2 \mathrm{~m}$ aperture and a large field. For the future, we intend to decrease these errors by employing unique dedicated reduction pipeline based on CPL procedures which uses UCAC-2 and UCAC-3 catalogs. These results are about two to three times better than those reported by other major surveys dedicated mainly to the discovery of new objects, which use much larger pixel scales and other observing protocols.

An important contribution could be obtained in the NEA research using $1 \mathrm{~m}$ class and even smaller telescopes, and this task was achieved during the last three years by the EURONEAR network, which used nine telescopes located in four countries in a team effort. Other runs are planned in the future on 1-2 m class telescopes to our specific objectives, while some funding continue to be searched in order to dedicate one or two 1-2 m class telescopes to the EURONEAR project.

Acknowledgements. The article is based on observations acquired in Pic du Midi, Haute Provence, La Silla, Cerro Tololo, Las Campanas, Cerro Armazones, Bucharest Urseanu, and York University Observatories. The run of Alex Tudorica in Cerro Tololo was supported by the University of Bucharest, Faculty of Physics, Romania. We thank Prof. Mircea Rusu from the University of Bucharest, Faculty of Physics, for his contribution. The runs of F. Pozo N., and A. Barr D. in Las Campanas, and C.P. Soto in La Silla were supported by the Universidad Católica del Norte. The EURONEAR website is hosted by the Institut de Mécanique Céleste et de Calcul des Ephémérides, Observatoire de Paris, France. To analyze the photometry data we used the ALC software (Pravec, private communication). Observations at SAAO were done by Henry Hsieh in a joint proposal including Alan Fitzsimmons, Petr Pravec and Alan Harris. Adrian Galad was supported by the Slovak Grant Agency for Science VEGA (Grant 2/0016/09) and the Grant Agency of the Czech Republic (Grant 205/09/1107).

\section{References}

Bec-Borsenberger, A., \& Rocher, P. 1988, in Dévelopments Récents en Planétologie Dynamique, 29

Bernardi, F., Boattini, A., D’Abramo, G., et al. 2002, Asteroids, Comets, and Meteors: ACM 2002, ed. B. Warmbein, in ESA SP-500, 801

Boattini, A., D'Abramo, G., Scholl, H., et al. 2004, A\&A, 418, 743

Boattini, A., D'Abramo, G., Valsecchi, G. B., et al. 2007, Earth Moon and Planets, 100, 259

Bowell, E., \& Muinonen, K. 1994, in Hazards Due to Comets and Asteroids, ed. T. Gehrels, M. S. Matthews, \& A. M. Schumann, 149

Buie, M. W., Parker, J. W., Wittman, D., et al. 2008, Minor Planet Circulars, 6312,3 
M. Birlan et al.: More than 160 NEAs in the EURONEAR network

Cavadore, C., Colas, F., Vaduvescu, O., et al. 2006, Minor Planet Circulars, 5711, 2

Cavadore, C., Elst, E. W., Lagerkvist, C.-I., et al. 2008, Minor Planet Circulars, 6336, 9

Devyatkin, A. V., Kulish, A. P., Kouprianov, V. V., et al. 2007, in IAU Symp. 236, ed. G. B. Valsecchi, \& D. Vokrouhlický, 391

Elst, E. W., Lagerkvist, C.-I., Boattini, A., Boehnhardt, H., \& Vaduvescu, O. 2008a, Minor Planet Circulars, 6287, 8

Elst, E. W., Lagerkvist, C.-I., Boattini, A., et al. 2008b, Minor Planet Circulars, 6257,3

Elst, E. W., Lagerkvist, C.-I., Boattini, A., et al. 2008c, Minor Planet Circulars, 6312,5

Everhart, E. 1985, in Dynamics of Comets: Their Origin and Evolution, ed. A. Carusi, \& G. B. Valsecchi (Dordrecht: Reidel), Astrophysics and Space Science Library, 115, Proc. Colloq., held in Rome, Italy, June 11-15, 1984, 83,185

Gehrels, T., \& Jedicke, R. 1996, Earth Moon and Planets, 72, 233

Helin, E. F., Pravdo, S. H., Rabinowitz, D. L., \& Lawrence, K. J. 1997, New York Academy Sciences Annals, 822, 6

Howell, S. B., Koehn, B., Bowell, E., \& Hoffman, M. 1996, AJ, 112, 1302

Ivantsov, A. V., Aslan, Z., Gumerov, R. I., et al. 2008, in American Institute of Physics Conf. Ser., 1043, 167

Kern, S. D., Sheppard, S. S., Vaduvescu, O., \& Schechter, P. L. 2008, Minor Planet Circulars, 6336, 10

Lainey, V. 2008, Planet. Space Sci., 56, 1766

Lainey, V., \& Vienne, A. 2009, in Connaissance des Temps (EDP Sciences), 177

Lainey, V., Dehant, V., \& Pätzold, M. 2007, A\&A, 465, 1075

Larson, S., Spahr, T., Brownlee, J., Hergenrother, C., \& McNaught, R. 1999, In Proceedings of the 1999 AMOS Technical Conference, 182

Milani, A., Chesley, S. R., Boattini, A., \& Valsecchi, G. B. 2000a, Icarus, 145, 12

Milani, A., Chesley, S. R., \& Valsecchi, G. B. 2000b, Planet. Space Sci., 48, 945

Milani, A., Chesley, S. R., Chodas, P. W., \& Valsecchi, G. B. 2002, Asteroids III, 55

Milani, A., Gronchi, G. F., \& Knežević, Z. 2007, Earth Moon and Planets, 100, 83

Morbidelli, A., Bottke, Jr., W. F., Froeschlé, C., \& Michel, P. 2002, Asteroids III, 409
Morrison, D. 1992, Mercury, 21, 103

Pozo, F., Barr, A., Vaduvescu, O., et al. 2008, Minor Planet Electronic Circulars, 48

Pravec, P., \& Hahn, G. 1997, Icarus, 127, 431

Rocher, P. 2007, in Notes scientifiques et techniques de 1'IMCCE, ISBN: 2910015-54-8

Scotti, J. V., Pozo, F., Barr, A., et al. 2008a, Minor Planet Electronic Circulars, 46

Scotti, J. V., Pozo, F., Barr, A., et al. 2008b, Minor Planet Electronic Circulars, 45

Sheppard, S. S., Vaduvescu, O., Galad, A., et al. 2008, Minor Planet Circulars, 6225,1

Stokes, G. H., Evans, J. B., \& Larson, S. M. 2002, Asteroids III, 45

Tholen, D. J., Bernardi, F., \& Micheli, M. 2008a, in AAS/Division for Planetary Sciences Meeting Abstracts, 40, 27.02

Tholen, D. J., Elst, E. W., Lagerkvist, C.-I., et al. 2008b, Minor Planet Circulars, 6226,5

Tichá, J., Tichý, M., \& Kočer, M. 2002, in Asteroids, Comets, and Meteors: ACM 2002, ed. B. Warmbein, ESA SP-500, 793

Tichá, J., Tichý, M., \& Moravec, Z. 2000, Planet. Space Sci., 48, 787

Tubbiolo, A. F., Bressi, T. H., Gilmore, A. C., et al. 2008, Minor Planet Electronic Circulars, 66

Čapek, D., \& Vokrouhlický, D. 2004, Icarus, 172, 526

Vaduvescu, O., \& Tudorica, A. 2008, Minor Planet Circulars, 6312, 6

Vaduvescu, O., Colque, J. P., \& Nedelcu, A. 2007a, Minor Planet Circulars, 6143,11

Vaduvescu, O., Nedelcu, A., Birlan, M., \& Sonka, A. 2007b, Minor Planet Circulars, 5986, 3

Vaduvescu, O., Nedelcu, A., Lombardi, G., et al. 2007c, Minor Planet Electronic Circulars, 32

Vaduvescu, O., Nedelcu, A., \& Sonka, A. 2007d, Minor Planet Circulars, 6008, 5

Vaduvescu, O., Birlan, M., Colas, F., Sonka, A., \& Nedelcu, A. 2008, Planet. Space Sci., 56, 1913

Viggh, H. E. M., Stokes, G. H., Shelly, F. C., Blythe, M. S., \& Stuart, J. S. 1998, in Space 98, ed. R. G. Galloway, \& S. Lokaj, 373

Young, J., Vaduvescu, O., Tudorica, A., et al. 2008, Minor Planet Electronic Circulars, 63 


\section{Appendix A}

Table A.1. The observing log file.

\begin{tabular}{|c|c|c|c|c|c|c|c|c|}
\hline Observatory & Asteroid & Class & Date (UT) & App mag (V) & Exp time & $\mathrm{Nr}$ pos & $\mu$ & Obs-Dis \\
\hline \multirow[t]{14}{*}{ Pic du Midi } & 2006 HV 57 & NEA & 2006 May 16 & 19.2 & 300 & 10 & 0.5 & $18 \mathrm{~d}$ \\
\hline & $2006 \mathrm{JU}$ & NEA & 2006 May 16 & 19.6 & 300 & 10 & 0.9 & $13 \mathrm{~d}$ \\
\hline & 2006 JU41 & NEA & 2006 May 17 & 19.8 & 300 & 5 & 0.7 & $9 \mathrm{~d}$ \\
\hline & 2006 HV57 & NEA & 2006 May 18 & 19.3 & 300 & 8 & 0.5 & $20 d$ \\
\hline & (144898) 2004 VD17 & VI & 2006 May 18 & 19.7 & 180 & 11 & 0.9 & $2 y$ \\
\hline & $2006 \mathrm{JF} 42$ & PHA & 2006 May 18 & 18.2 & 120 & 10 & 2.1 & $7 d$ \\
\hline & (139211) 2001 GN2 & PHA & 2006 May 19 & 19.2 & 120 & 10 & 1.5 & $5 y$ \\
\hline & 2006 GW2 & NEA & 2006 May 19 & 19.2 & 300 & 10 & 0.1 & $40 \mathrm{~d}$ \\
\hline & 2006 HQ30 & PHA & 2006 May 21 & 19.0 & 240 & 9 & 1.6 & $30 d$ \\
\hline & 1997 XR2 & PHA & 2006 May 22 & 19.7 & 300 & 1 & 1.5 & $9 y$ \\
\hline & $2006 \mathrm{~KB} 1$ & NEA & 2006 May 23 & 18.5 & 120 & 4 & 2.8 & $2 d$ \\
\hline & 2006 KD1 & PHA & 2006 May 23 & 18.3 & 150 & 14 & 2.3 & $3 d$ \\
\hline & $2006 \mathrm{KC}$ & NEA & 2006 May 24 & 18.8 & 120 & 16 & 3.4 & $5 d$ \\
\hline & (154715) 2004 LB6 & NEA & 2006 May 24 & 19.5 & 300 & 4 & 0.4 & $2 y$ \\
\hline \multirow[t]{19}{*}{ Haute Provence } & (68950) 2002 QF15 & PHA & 2006 May 28 & 14.6 & 60 & 16 & 5.1 & $4 y$ \\
\hline & (68950) 2002 QF15 & PHA & 2006 May 30 & 14.7 & 60 & 11 & 4.6 & $4 y$ \\
\hline & 2007 FK 1 & NEA & 2007 May 06 & 16.7 & 120 & 12 & 5.3 & $50 \mathrm{~d}$ \\
\hline & 2007 FV42 & NEA & 2007 May 06 & 17.5 & 90 & 10 & 1.0 & $48 d$ \\
\hline & 2007 FL1 & NEA & 2007 May 07 & 17.9 & 90 & 10 & 3.5 & $51 d$ \\
\hline & 2007 DZ40 & NEA & 2007 May 08 & 18.0 & 90 & 5 & 3.3 & $76 \mathrm{~d}$ \\
\hline & (184990) 2006 KE89 & NEA & 2007 May 08 & 18.6 & 90 & 10 & 1.7 & $1 \mathrm{y}$ \\
\hline & $2006 \mathrm{~GB}$ & PHA & 2007 May 08 & 19.1 & 120 & 10 & 2.2 & $1 \mathrm{y}$ \\
\hline & 2007 DF8 & NEA & 2007 May 08 & 19.6 & 180 & 10 & 1.3 & $75 \mathrm{~d}$ \\
\hline & 2007 DT103 & PHA & 2007 May 08 & 19.7 & 300 & 2 & 0.3 & $71 d$ \\
\hline & 2007 HX4 & NEA & 2007 May 09 & 19.9 & 300 & 7 & 2.7 & $18 \mathrm{~d}$ \\
\hline & 2007 FE1 & NEA & 2007 May 09 & 19.8 & 180 & 11 & 1.4 & $51 d$ \\
\hline & $2007 \mathrm{JX} 2 *$ & PHA & 2007 May 09 & 19.0 & 60 & 11 & 4.6 & $1 d$ \\
\hline & 2006 HR29 & NEA & 2007 May 09 & 17.9 & 60 & 8 & 3.3 & $1 \mathrm{y}$ \\
\hline & 2007 HX82 & NEA & 2007 May 09 & 19.7 & 180 & 11 & 1.4 & $13 \mathrm{~d}$ \\
\hline & 2006 VY13 & NEA & 2007 May 10 & 19.7 & 300 & 4 & 0.6 & $1 y$ \\
\hline & 2007 CQ5 & NEA & 2007 May 10 & 19.3 & 180 & 7 & 1.4 & $93 d$ \\
\hline & 2007 JW2 & NEA & 2007 May 10 & 19.1 & 180 & 10 & 1.8 & $3 d$ \\
\hline & 2006 WL3 & NEA & 2007 May 10 & 19.8 & 300 & 6 & 1.8 & $1 \mathrm{y}$ \\
\hline \multirow[t]{6}{*}{ Cerro Armazones } & 2007 VD12 & PHA & 2007 Nov. 17 & 16.4 & 60 & 19 & 2.7 & $12 d$ \\
\hline & 2007 VY7 & NEA & 2007 Nov. 17 & 17.9 & 120 & 10 & 1.8 & $7 d$ \\
\hline & 2006 US216 & PHA & 2007 Nov. 17 & 18.5 & 120 & 14 & 2.7 & $1 \mathrm{y}$ \\
\hline & 2007 TD71 & PHA & 2007 Nov. 18 & 19.5 & 120 & 4 & 3.6 & $36 \mathrm{~d}$ \\
\hline & 2007 GS3 & PHA & 2007 Nov. 18 & 18.1 & 90 & 18 & 2.8 & $1 \mathrm{y}$ \\
\hline & 2007 VT6 & PHA & 2007 Nov. 18 & 18.6 & 60 & 8 & 3.2 & $15 \mathrm{~d}$ \\
\hline \multirow[t]{18}{*}{ Cerro Tololo } & 2008 JZ7 & NEA & 2008 May 30 & 19.4 & 60 & 11 & 2.0 & $25 d$ \\
\hline & $2008 \mathrm{JO}$ & NEA & 2008 May 30 & 19.6 & 60 & 7 & 2.4 & $28 d$ \\
\hline & 2008 HF2 & NEA & 2008 May 30 & 19.0 & 30 & 8 & 5.2 & $34 d$ \\
\hline & 2008 FS6 & NEA & 2008 May 30 & 19.4 & 60 & 5 & 0.7 & $64 d$ \\
\hline & 2008 DY & NEA & 2008 May 30 & 19.8 & 60 & 10 & 1.4 & $66 \mathrm{~d}$ \\
\hline & 2008 JS26 & NEA & 2008 Мay 30 & 18.9 & 30 & 10 & 6.7 & $16 \mathrm{~d}$ \\
\hline & 2008 JU2 & NEA & 2008 May 30 & 18.9 & 30 & 12 & 7.1 & $27 d$ \\
\hline & 2007 XH16 & PHA & 2008 May 30 & 19.3 & 60 & 10 & 2.7 & $1 y$ \\
\hline & 2008 JW2 & PHA & 2008 May 30 & 19.1 & 60 & 10 & 1.4 & $27 d$ \\
\hline & 2007 YB2 & NEA & 2008 May 30 & 20.0 & 120 & 8 & 0.7 & $1 \mathrm{y}$ \\
\hline & 2008 KE6 * & NEA & 2008 May 30 & 19.9 & 30 & 6 & 4.7 & $1 d$ \\
\hline & 2008 JA8 * & NEA & 2008 May 30 & 19.0 & 30 & 11 & 3.5 & $25 d$ \\
\hline & 2008 EZ97 & NEA & 2008 May 30 & 18.1 & 30 & 11 & 2.0 & $76 d$ \\
\hline & 2008 JY30 & NEA & 2008 May 30 & 18.4 & 20 & 9 & 9.4 & $16 \mathrm{~d}$ \\
\hline & $2008 \mathrm{DE}$ & PHA & 2008 May 30 & 18.0 & 20 & 10 & 2.3 & $77 d$ \\
\hline & 2007 WV4 & PHA & 2008 May 30 & 19.5 & 30 & 3 & 4.4 & $1 y$ \\
\hline & 2008 FN6 & NEA & 2008 May 31 & 19.1 & 30 & 10 & 4.9 & $61 d$ \\
\hline & 2008 CP23 & NEA & 2008 May 31 & 19.8 & 60 & 4 & 2.3 & $120 \mathrm{~d}$ \\
\hline
\end{tabular}


Table A.1. continued.

\begin{tabular}{|c|c|c|c|c|c|c|c|c|}
\hline \multirow[t]{7}{*}{ Observatory } & Asteroid & Class & Date (UT) & App mag (V) & Exp time & $\mathrm{Nr}$ pos & $\mu$ & Obs-Dis \\
\hline & $2008 \mathrm{KV} 2$ & NEA & 2008 May 31 & 18.3 & 15 & 10 & 4.7 & $3 \mathrm{~d}$ \\
\hline & 2008 KF6 & VI & 2008 May 31 & 18.6 & 15 & 10 & 9.1 & $1 d$ \\
\hline & 2008 KW2 & NEA & 2008 May 31 & 18.1 & 15 & 9 & 5.3 & $3 d$ \\
\hline & $2008 \mathrm{KN} 11 *$ & VI & 2008 May 31 & 20.4 & 60 & 12 & 1.1 & $1 d$ \\
\hline & 2008 EC69 & PHA & 2008 May 31 & 17.1 & 20 & 9 & 5.4 & $100 d$ \\
\hline & $2008 \mathrm{JJ}$ & NEA & 2008 Jun. 01 & 16.2 & 10 & 2 & 11.6 & $32 \mathrm{~d}$ \\
\hline \multirow[t]{54}{*}{ Las Campanas } & 1994 AW1 & PHA & 2008 Mar. 08 & 18.0 & 60 & 49 & 1.2 & $14 y$ \\
\hline & 2005 TJ174 & NEA & 2008 Jun. 04 & 19.0 & 120 & 5 & 0.8 & $3 y$ \\
\hline & 2008 KB12 & NEA & 2008 Jun. 05 & 18.5 & 20 & 8 & 7.3 & $7 \mathrm{~d}$ \\
\hline & 2005 UY5 & NEA & 2008 Jun. 06 & 18.7 & 60 & 8 & 0.7 & $3 y$ \\
\hline & 2008 TC4 & NEA & 2008 Oct. 18 & 18.1 & 15 & 15 & 9.9 & $12 \mathrm{~d}$ \\
\hline & 2008 RS26 & NEA & 2008 Oct. 18 & 19.5 & 150 & 10 & 0.7 & $46 d$ \\
\hline & 2008 KZ5 & PHA & 2008 Oct. 18 & 18.4 & 45 & 16 & 3.6 & $142 d$ \\
\hline & 2008 TX3 & NEA & 2008 Oct. 18 & 18.9 & 30 & 16 & 4.4 & $12 \mathrm{~d}$ \\
\hline & 2001 SG10 & PHA & 2008 Oct. 18 & 19.0 & 60 & 16 & 1.9 & $7 y$ \\
\hline & (190491) 2000 FJ10 & NEA & 2008 Oct. 18 & 17.0 & 15 & 16 & 6.1 & $8 y$ \\
\hline & 2007 TA19 & PHA & 2008 Oct. 18 & 19.6 & 45 & 16 & 2.8 & $1 \mathrm{y}$ \\
\hline & (171576) 1999 VP11 & PHA & 2008 Oct. 18 & 17.4 & 15 & 11 & 7.3 & $9 y$ \\
\hline & 2006 WR1 & NEA & 2008 Oct. 19 & 19.8 & 45 & 15 & 2.8 & $2 y$ \\
\hline & 2005 UH6 & PHA & 2008 Oct. 19 & 19.7 & 30 & 15 & 3.9 & $3 y$ \\
\hline & 2008 QT3 & PHA & 2008 Oct. 19 & 19.2 & 150 & 10 & 0.1 & $54 \mathrm{~d}$ \\
\hline & 2008 TD4 & NEA & 2008 Oct. 19 & 19.0 & 50 & 15 & 2.5 & $12 \mathrm{~d}$ \\
\hline & 2008 TN26 & NEA & 2008 Oct. 19 & 19.9 & 90 & 12 & 2.2 & $10 d$ \\
\hline & 2008 SR1 & NEA & 2008 Oct. 19 & 19.9 & 180 & 15 & 0.3 & $26 d$ \\
\hline & $2008 \mathrm{QZ}$ & NEA & 2008 Oct. 19 & 19.3 & 180 & 10 & 0.2 & $58 d$ \\
\hline & 2008 SP7 & NEA & 2008 Oct. 19 & 18.5 & 60 & 15 & 1.3 & $26 d$ \\
\hline & 2008 SJ82 & NEA & 2008 Oct. 19 & 19.9 & 90 & 14 & 1.6 & $23 d$ \\
\hline & 2008 MH1 & NEA & 2008 Oct. 22 & 19.3 & 40 & 15 & 2.6 & $119 d$ \\
\hline & 2008 OB9 & PHA & 2008 Oct. 22 & 20.6 & 200 & 15 & 0.5 & $89 d$ \\
\hline & 2008 CR118 & PHA & 2008 Oct. 22 & 19.8 & 45 & 10 & 2.0 & $245 d$ \\
\hline & 2008 RG98 & NEA & 2008 Oct. 22 & 19.2 & 90 & 9 & 1.5 & $42 \mathrm{~d}$ \\
\hline & 2008 SE85 & NEA & 2008 Oct. 22 & 19.6 & 30 & 10 & 4.8 & $24 d$ \\
\hline & 2008 TV25 & NEA & 2008 Oct. 22 & 18.7 & 30 & 10 & 4.4 & $13 d$ \\
\hline & 2008 SU1 & NEA & 2008 Oct. 23 & 18.5 & 20 & 6 & 5.2 & $30 d$ \\
\hline & 2008 SX7 & NEA & 2008 Oct. 23 & 19.4 & 60 & 7 & 2.1 & $32 d$ \\
\hline & 2008 SW150 & NEA & 2008 Oct. 23 & 20.1 & 60 & 7 & 2.2 & $24 d$ \\
\hline & $2008 \mathrm{UC}$ & NEA & 2008 Oct. 23 & 19.1 & 60 & 8 & 0.4 & $34 d$ \\
\hline & 2008 TF4 & NEA & 2008 Oct. 23 & 19.2 & 50 & 8 & 2.2 & $17 d$ \\
\hline & 2008 RE80 & NEA & 2008 Oct. 23 & 20.2 & 120 & 6 & 0.8 & $42 \mathrm{~d}$ \\
\hline & 2008 TE 157 & NEA & 2008 Oct. 23 & 20.2 & 100 & 8 & 0.9 & $21 d$ \\
\hline & 2008 TJ157 & NEA & 2008 Oct. 23 & 20.6 & 120 & 6 & 1.1 & $17 d$ \\
\hline & 2008 TK 157 & NEA & 2008 Oct. 23 & 19.2 & 60 & 8 & 0.5 & $30 d$ \\
\hline & 2008 TA1 & NEA & 2008 Oct. 23 & 20.1 & 120 & 6 & 1.4 & $21 d$ \\
\hline & 2008 RP108 & NEA & 2008 Oct. 23 & 20.0 & 120 & 6 & 0.2 & $43 d$ \\
\hline & 2008 SX245 & NEA & 2008 Oct. 23 & 19.4 & 60 & 8 & 1.1 & $24 d$ \\
\hline & 2008 SS251 & NEA & 2008 Oct. 23 & 20.3 & 120 & 8 & 0.2 & $27 d$ \\
\hline & 2008 QD1 & NEA & 2008 Oct. 23 & 18.7 & 90 & 6 & 0.9 & $60 d$ \\
\hline & 2008 SO1 & NEA & 2008 Oct. 23 & 19.8 & 45 & 6 & 3.1 & $31 d$ \\
\hline & 2008 SV7 & NEA & 2008 Oct. 23 & 19.9 & 60 & 7 & 2.4 & $29 d$ \\
\hline & 2008 SQ7 & NEA & 2008 Oct. 23 & 19.4 & 30 & 4 & 4.4 & $30 d$ \\
\hline & 2008 UT2 * & NEA & 2008 Oct. 24 & 20.0 & 20 & 5 & 4.9 & $1 d$ \\
\hline & 2008 UR2 * & NEA & 2008 Oct. 24 & 19.8 & 40 & 8 & 3.7 & $1 d$ \\
\hline & 2002 TW55 & PHA & 2008 Oct. 24 & 18.7 & 20 & 9 & 7.1 & $6 y$ \\
\hline & 2001 SG10 & PHA & 2008 Oct. 24 & 19.1 & 60 & 10 & 1.5 & $7 y$ \\
\hline & 2005 WK4 & PHA & 2008 Oct. 24 & 19.5 & 60 & 10 & 1.8 & $3 y$ \\
\hline & $2001 \mathrm{KO} 20$ & NEA & 2008 Oct. 24 & 20.0 & 60 & 10 & 1.0 & $7 y$ \\
\hline & 2008 TT26 & NEA & 2008 Oct. 24 & 15.2 & 3 & 5 & 41.0 & $15 d$ \\
\hline & 2001 UY4 & PHA & 2008 Oct. 24 & 16.8 & 10 & 10 & 8.8 & $7 y$ \\
\hline & 2008 MB5 & NEA & 2008 Oct. 24 & 20.2 & 80 & 9 & 1.6 & $114 \mathrm{~d}$ \\
\hline & 2008 RT26 & NEA & 2008 Oct. 24 & 20.1 & 80 & 8 & 1.5 & $47 d$ \\
\hline
\end{tabular}


Table A.1. continued.

\begin{tabular}{|c|c|c|c|c|c|c|c|c|}
\hline Observatory & Asteroid & Class & Date (UT) & App mag (V) & Exp time & $\mathrm{Nr}$ pos & $\mu$ & Obs-Dis \\
\hline \multirow{2}{*}{ La Silla $1 \mathrm{~m}$} & 2007 PA8 & VI & 2007 Aug. 13 & 19.2 & 120 & 4 & 0.8 & $4 d$ \\
\hline & 2007 FV42 & NEA & 2007 Aug. 13 & 16.5 & 120 & 12 & 0.4 & $1 \mathrm{y}$ \\
\hline \multirow[t]{16}{*}{ La Silla $2.2 \mathrm{~m}$} & 2008 EA8 & VI & 2008Mar. 11 & 21.4 & 60 & 14 & 2.7 & $5 d$ \\
\hline & 2008 EX5 & VI & 2008 Mar. 11 & 20.3 & 60 & 15 & 2.4 & $7 d$ \\
\hline & 2008 EM7 & VI & 2008 Mar. 11 & 20.6 & 60 & 15 & 3.1 & $5 d$ \\
\hline & 2008 ER7 & VI & 2008 Mar. 11 & 20.7 & 60 & 13 & 1.8 & $5 d$ \\
\hline & 2005 GE59 & PHA & 2008 Mar. 11 & 20.6 & 60 & 14 & 0.8 & $3 y$ \\
\hline & (162173) 1999 JU3 & PHA & 2008 Mar. 12 & 17.9 & 60 & 15 & 3.4 & $9 y$ \\
\hline & 2008 EL6 & PHA & 2008 Mar. 12 & 17.2 & 10 & 17 & 15 & $7 \mathrm{~d}$ \\
\hline & (162173) 1999 JU3 & PHA & 2008 Mar. 13 & 17.9 & 60 & 8 & 3.4 & $9 y$ \\
\hline & 2006 GZ & NEA & 2008 Mar. 13 & 21.3 & 120 & 8 & 0.9 & $2 y$ \\
\hline & 2008 DA4 & VI & 2008 Mar. 13 & 22.0 & 120 & 8 & 1.0 & $2 y$ \\
\hline & 2008 CE6 & NEA & 2008 Mar. 13 & 21.2 & 120 & 8 & 0.8 & $33 d$ \\
\hline & 2008 DL5 & PHA & 2008 Mar. 13 & 19.7 & 30 & 8 & 4.0 & $13 \mathrm{~d}$ \\
\hline & 2008 EJ9 & NEA & 2008 Mar. 13 & 20.0 & 60 & 7 & 5.2 & $4 d$ \\
\hline & 2008 EQ7 & NEA & 2008 Mar. 13 & 20.1 & 60 & 5 & 2.4 & $7 d$ \\
\hline & 2008 DJ5 & NEA & 2008 Mar. 13 & 20.3 & 60 & 5 & 1.5 & $13 \mathrm{~d}$ \\
\hline & 2008 EA9 & VI & 2008 Mar. 13 & 21.0 & 60 & 5 & 2.4 & $7 \mathrm{~d}$ \\
\hline \multirow[t]{25}{*}{ Urseanu } & (1980) Tezcatlipoca & NEA & 2006 Nov. 07 & 13.7 & 15 & 3 & 3.0 & $56 y$ \\
\hline & (5143) Heracles & NEA & 2006 Nov. 13 & 14.5 & 15 & 3 & 2.4 & $53 y$ \\
\hline & 2007 PU11 & NEA & 2007 Oct. 16 & 15.1 & 15 & 7 & 2.2 & $18 \mathrm{y}$ \\
\hline & 2007 TS19 & PHA & 2007 Oct. 16 & 16.0 & 15 & 4 & 9.2 & $6 \mathrm{~d}$ \\
\hline & (4954) Eric & NEA & 2007 Nov. 02 & 12.3 & 15 & 3 & 2.1 & $32 y$ \\
\hline & (3200) Phaethon & PHA & 2007 Nov. 28 & 14.0 & 15 & 3 & 5.2 & $24 y$ \\
\hline & (1036) Ganymed & NEA & 2007 Nov. 29 & 13.4 & 15 & 3 & 0.6 & $83 y$ \\
\hline & (3288) Seleucus & NEA & 2008 Jan. 28 & 15.7 & 15 & 3 & 1.0 & $26 y$ \\
\hline & 2007 TU24 & PHA & 2008 Jan. 30 & 11.6 & 15 & 3 & 36.7 & $90 \mathrm{~d}$ \\
\hline & (1620) Geographos & PHA & 2008 Feb. 25 & 12.8 & 15 & 3 & 3.6 & $57 y$ \\
\hline & 1998 YP11 (11398) & NEA & 2008 Mar. 23 & 14.7 & 15 & 7 & 3.3 & $25 y$ \\
\hline & (1685) Toro & NEA & 2008 Mar. 03 & 14.7 & 15 & 4 & 2.1 & $60 y$ \\
\hline & (170891) 2004 TY16 & NEA & 2008 Mar. 03 & 15.9 & 15 & 4 & 2.1 & $4 y$ \\
\hline & 2008 CL1 & NEA & 2008 Mar. 09 & 16.1 & 15 & 4 & 10.2 & $34 d$ \\
\hline & (179806) 2002 TD66 & PHA & 2008 Mar. 09 & 16.6 & 15 & 3 & 4.2 & $6 y$ \\
\hline & 2005 NB7 & PHA & 2008 Apr. 20 & 14.8 & 15 & 4 & 15.8 & $3 y$ \\
\hline & (137170) 1999 HF1 & NEA & 2008 Apr. 20 & 15.4 & 15 & 4 & 1.9 & $9 y$ \\
\hline & (35107) $1991 \mathrm{VH}$ & PHA & 2008 May 01 & 16.8 & 15 & 7 & 1.4 & $17 y$ \\
\hline & (53319) 1999 JM8 & PHA & 2008 May 01 & 15.8 & 15 & 7 & 3.3 & $9 y$ \\
\hline & 2008 HW1 & NEA & 2008 May 05 & 16.7 & 15 & 11 & 8.8 & $36 d$ \\
\hline & 2008 JT35 & PHA & 2008 Jun. 30 & 16.0 & 10 & 5 & 11.4 & $55 \mathrm{~d}$ \\
\hline & (185851) 2000 DP107 & PHA & 2008 Oct. 01 & 15.1 & 15 & 3 & 3.2 & $8 y$ \\
\hline & (164400) 2005 GN59 & PHA & 2008 Oct. 03 & 14.1 & 15 & 3 & 14.2 & $3 y$ \\
\hline & (137032) 1998 UO1 & NEA & 2008 Oct. 03 & 14.0 & 15 & 4 & 10.2 & $10 y$ \\
\hline & (16960) 1998 QS52 & PHA & 2008 Oct. 10 & 14.3 & 15 & 6 & 4.8 & $10 y$ \\
\hline \multirow[t]{4}{*}{ York University } & (35396) 1997 XF11 & PHA & 2002 Nov. 09 & 13.8 & 20 & 23 & 7.6 & $5 y$ \\
\hline & (65803) Didymos & PHA & 2003 Dec. 04 & 13.7 & 60 & 35 & 2.7 & $7 y$ \\
\hline & (4179) Toutatis & PHA & 2004 Aug. 22 & 13.1 & 50 & 20 & 0.4 & $70 y$ \\
\hline & (4179) Toutatis & PHA & 2004 Sep. 12 & 11.9 & 30 & 20 & 1.2 & $70 \mathrm{y}$ \\
\hline
\end{tabular}

Notes. For each telescope location, the name of asteroid, its type, the date of observations, the apparent magnitude, the exposure time and the number of observations are presented. The table contains also the apparent motion of the asteroid at the time-period (d-days, y-years) between its discovery and the EURONEAR observation. 
M. Birlan et al.: More than 160 NEAs in the EURONEAR network

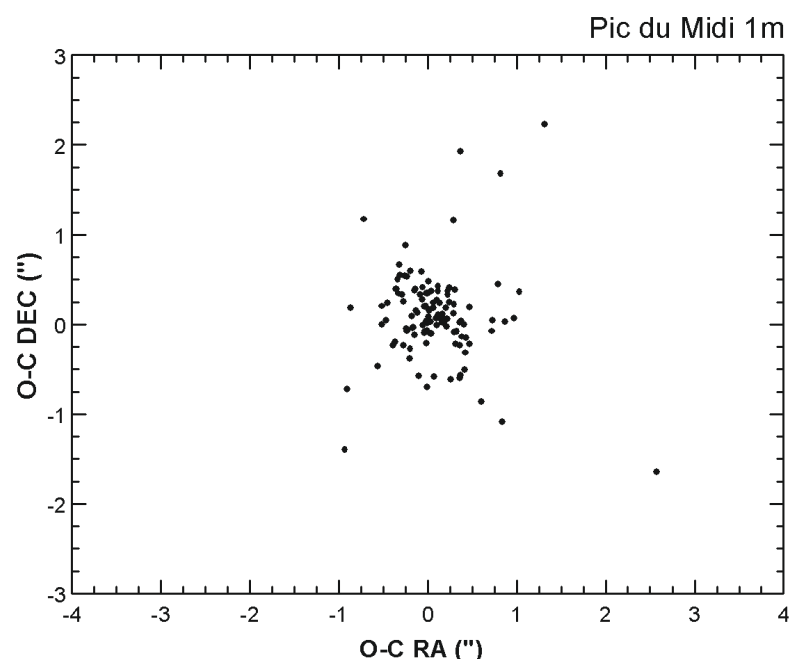

Fig. A.1. Pic du Midi $1 \mathrm{~m}$ : (o-c) residuals for 122 positions of 14 NEAs observed by EURONEAR in one run.

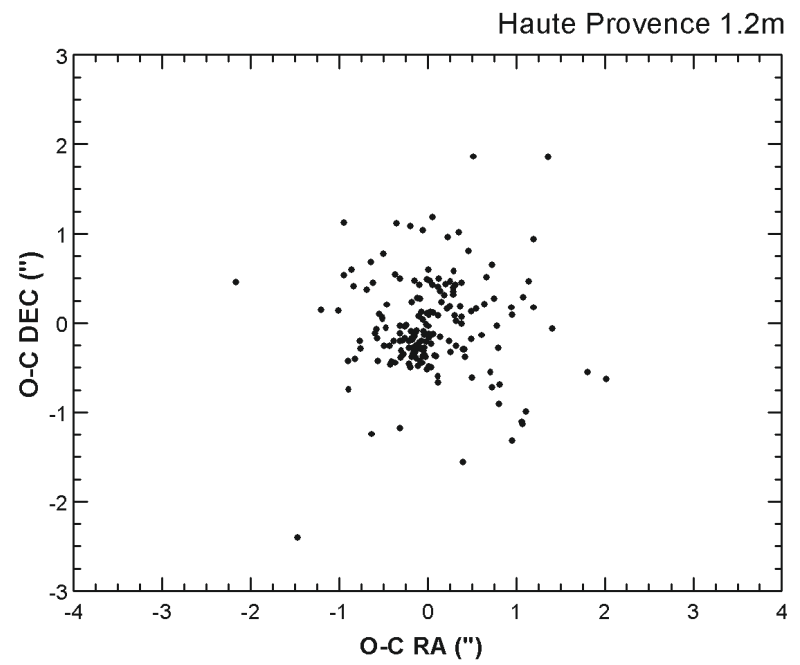

Fig. A.2. Haute Provence $1.2 \mathrm{~m}$ : (o-c) residuals for 171 positions of 19 NEAs observed by EURONEAR in one run.

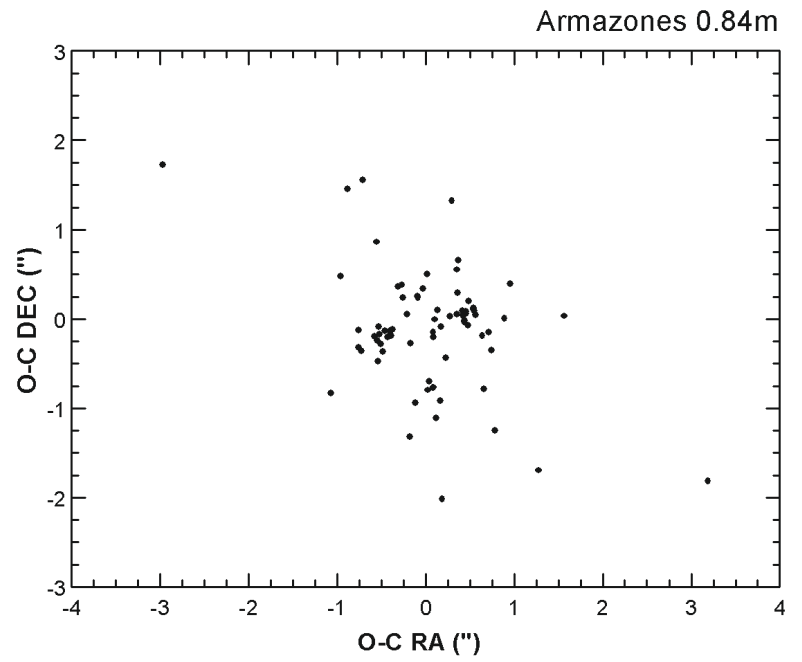

Fig. A.3. Armazones $0.84 \mathrm{~m}$ : (o-c) residuals for 73 positions of 6 NEAs observed by EURONEAR in one run.

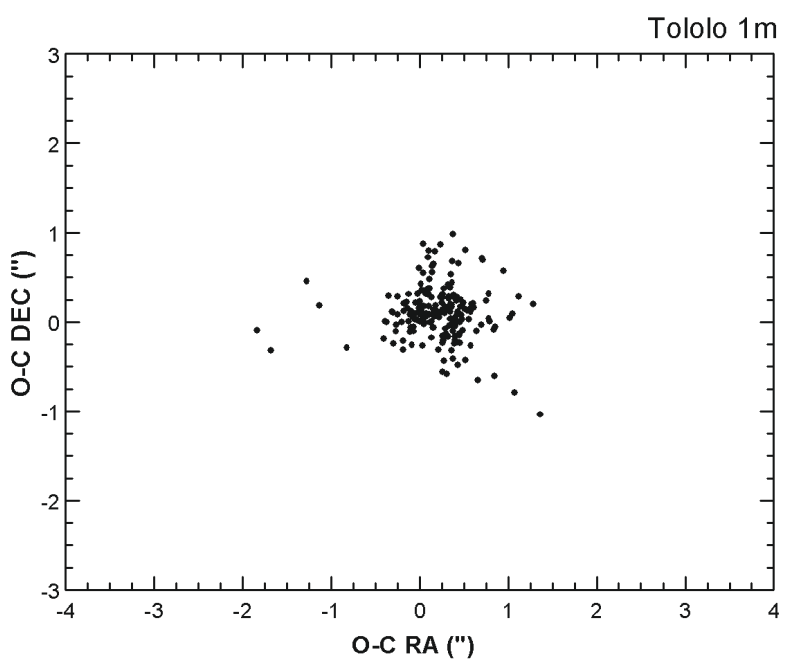

Fig. A.4. Tololo $1 \mathrm{~m}$ : (o-c) residuals for 207 positions of 24 NEAs observed by EURONEAR in one run.

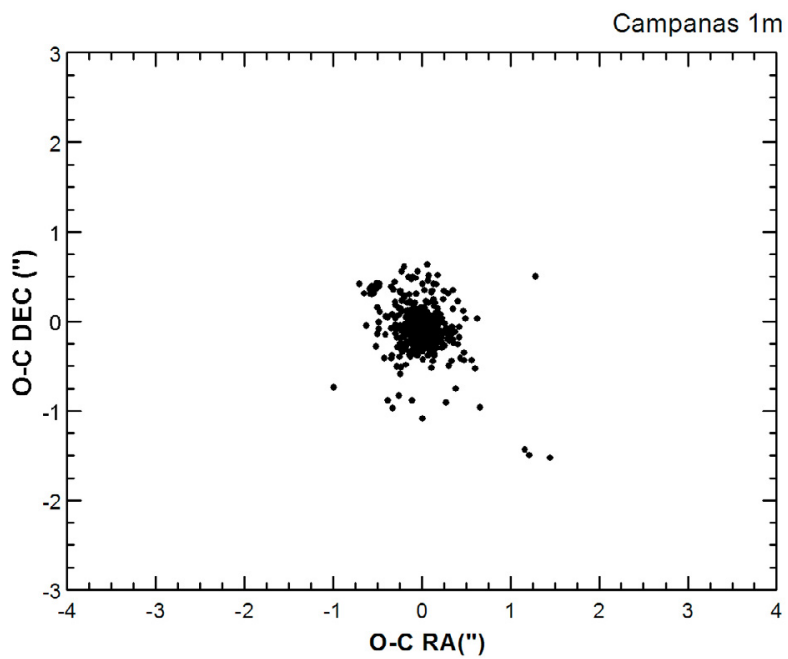

Fig. A.5. Campanas $1 \mathrm{~m}$ : (o-c) residuals for 575 positions of 54 NEAs observed by EURONEAR in three runs.

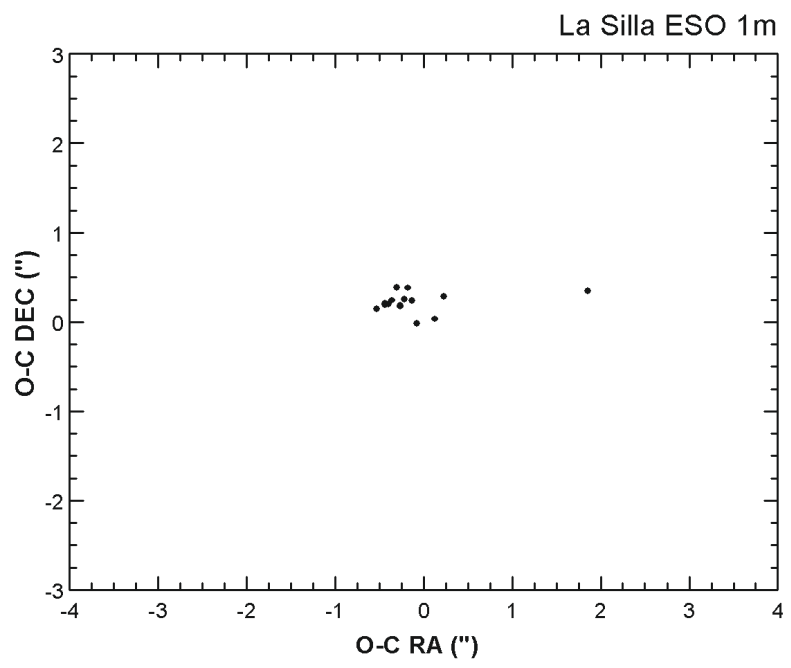

Fig. A.6. La Silla $1 \mathrm{~m}$ : (o-c) residuals for 16 positions of 2 NEAs observed by EURONEAR in one run. 
La Silla $2.2 \mathrm{~m}$

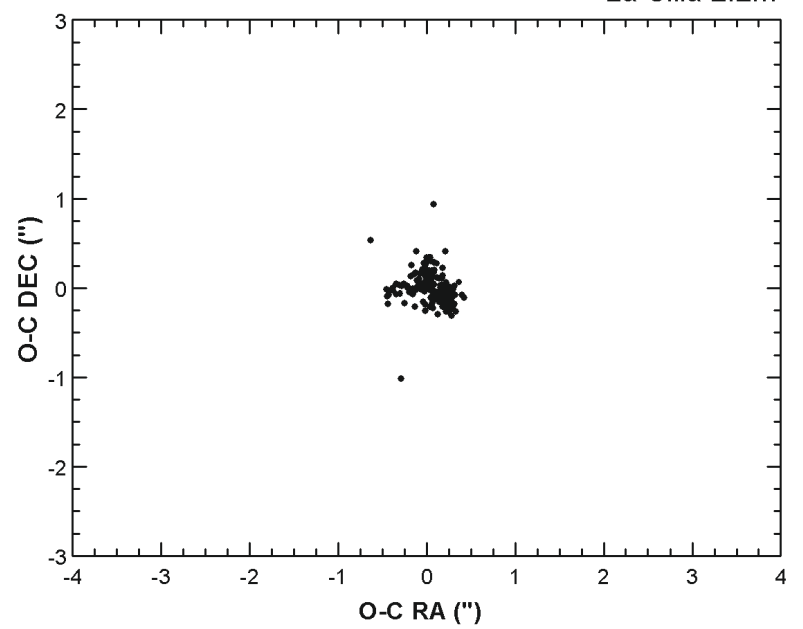

Fig. A.7. La Silla $2.2 \mathrm{~m}$ : (o-c) residuals for 165 positions of 15 NEAs observed by EURONEAR in one run.

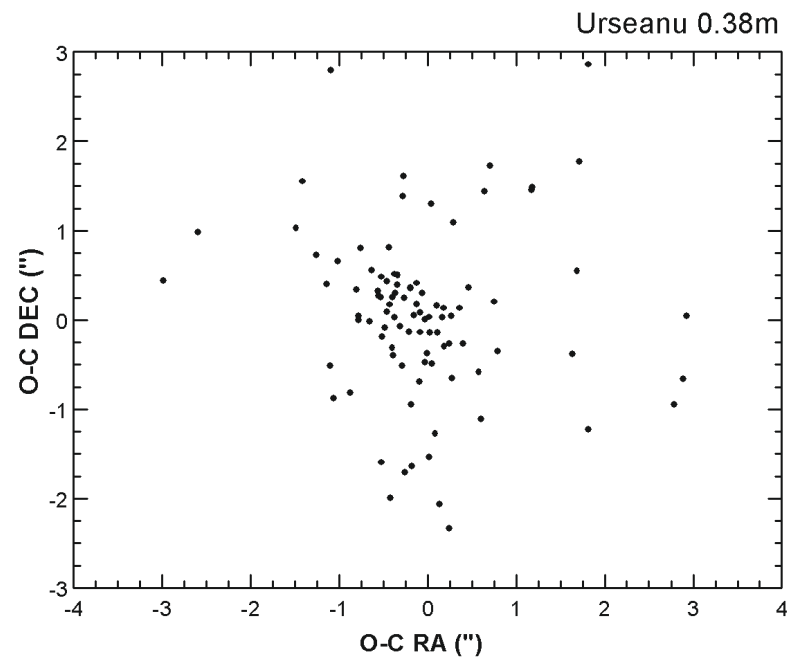

Fig. A.8. Urseanu $0.38 \mathrm{~m}$ : (o-c) residuals for 95 positions of 21 NEAs observed by EURONEAR in 18 runs.

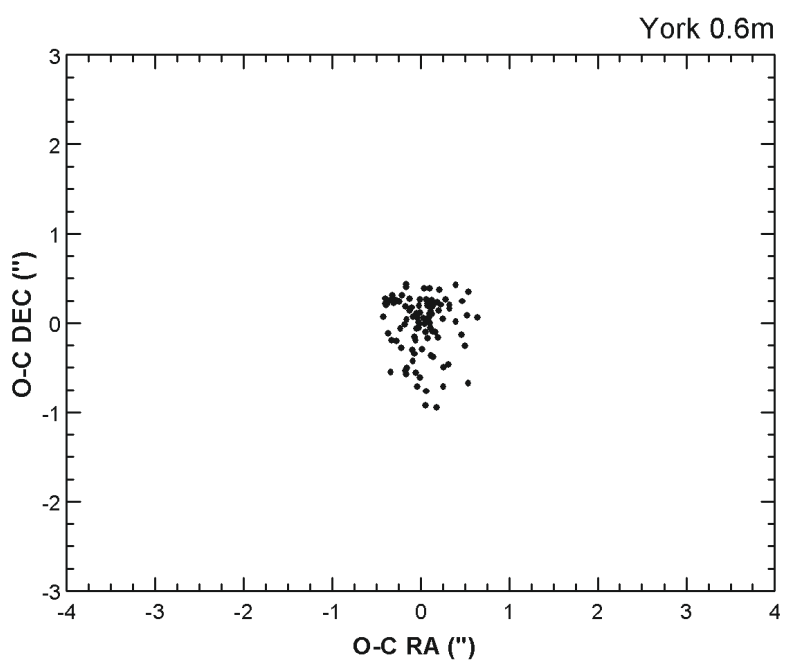

Fig. A.9. York University $0.6 \mathrm{~m}$ : (o-c) residuals for 98 positions of 4 NEAs observed by EURONEAR in 4 runs. 